\title{
Geschlecht, Parteizugehörigkeit und strategische Überlegungen
}

\author{
Die Einstellung weiblicher und männlicher Ratsmitglieder in \\ Baden-Württemberg zu lokalen Beteiligungsverfahren
}

\section{Florian Ruf}

Eingegangen: 18. April 2021 / Überarbeitet: 14. November 2021 / Angenommen: 17. November 2021 / Online publiziert: 15. Dezember 2021

(C) Der/die Autor(en) 2021

Zusammenfassung Ratsmitglieder sind zentrale Gatekeeper bei politischen Entscheidungen auf der lokalen Ebene. Dieser Aufsatz erklärt Unterschiede in den Einstellungen von 1638 weiblichen und männlichen Ratsmitgliedern zu repräsentativen, partizipativen und direktdemokratischen Beteiligungsformen. Er legt dabei einen Schwerpunkt auf (a) ihre Parteizugehörigkeit und (b) ihre strategischen Überlegungen, die sie anstrengen, um ihre Präferenzen durchzusetzen. Im Gegensatz zu den vorhandenen vergleichenden Studien legt diese Analyse einen Schwerpunkt auf die Unterschiede in den Einstellungen zwischen den Geschlechtern. Dabei wird gezeigt, dass die Einstellungen von weiblichen Ratsmitgliedern eben auch von ihren unterschiedlichen Erfahrungen und Perspektiven abhängen, die sie mit institutionellen Machtungleichgewichten machen. Oft befinden sich Frauen in einer Minderheitenposition im Rat und müssen zusätzliche Hindernisse überwinden, bevor sie ihre Präferenzen genauso wie ihre männlichen Kollegen durchsetzen können. Die Untersuchung zeigt, dass Ratsfrauen hier partizipative Beteiligungsmöglichkeiten grundsätzlich besser bewerten als ihre männlichen Kollegen. Vor allem Ratsfrauen der Partei die Linke und der CDU unterscheiden sich am stärksten von ihren männlichen Parteikollegen im Rat. Und auch eine gängige Annahme aus der Forschung zur Einstellung von Ratsmitgliedern bei Beteiligungsmöglichkeiten muss für zumindest für Baden-Württemberg ausdifferenziert werden: Es sind die Männer im Rat, die aus einer Position der Stärke heraus ihre Einstellungen begründen, während die Zugehörigkeit zur Mehrheit im Rat keine Auswirkungen für Ratsfrauen auf ihre Einstellungen gegenüber den drei unterschiedlichen Beteiligungsmöglichkeiten hat.

Schlüsselwörter Geschlecht · Einstellungen · Beteiligungsverfahren · Politische Eliten · Parteien · Strategie $\cdot$ Machtungleichgewichte $\cdot$ Kommunale Ebene

Florian Ruf $(\bowtie)$

Albert-Ludwigs-Universität Freiburg, Freiburg, Deutschland

E-Mail: flo.m.ruf@gmail.com 


\title{
Gender, party affiliation and power tactical considerations
}

The attitudes of female and male council members in Baden-Württemberg towards local participation procedures

\begin{abstract}
Council members are key gatekeepers in political decision-making at the local level. This paper explains differences in the attitudes of 1638 female and male council members toward representative, participatory, and direct democratic forms of participation. It focuses on (a) their party affiliation and (b) the strategic considerations they engage in to enforce their preferences. In contrast to existing comparative studies, this analysis places an emphasis on gender differences in attitudes. In doing so, it demonstrates that the attitudes of female council members depend precisely on the different experiences and perspectives they have with institutional power imbalances. Often, women find themselves in a minority position on the council and must overcome additional obstacles before they can assert their preferences as much as their male counterparts. The study shows that women councillors generally rate participatory participation opportunities better than their male counterparts. In particular, councilwomen from the Left Party and the CDU differ most from their male party colleagues on the council. And a common assumption from research on the attitude of council members toward participation opportunities must also be differentiated, at least for Baden-Württemberg: It is the men on the council who justify their attitudes from a position of strength, while being part of the majority on the council has no effect for women councillors on their attitudes toward the three different participation opportunities.
\end{abstract}

Keywords Gender - Attitudes - Participation procedures - Political elites - Parties · Power and power imbalances $\cdot$ Local level

\section{Einleitung}

Männer dominieren Gemeinderäte in den deutschen Kommunen (Holtkamp et al. 2011; Magin 2011; Smith et al. 2012; Egner 2013a; Ruf 2019). Neben diesem aus Sicht der politischen Repräsentation (Pitkin 1967; Phillips 1994; Childs und Lovenduski 2013) offensichtlichsten gesellschaftspolitischen und demokratietheoretisch höchst problematischen geschlechtsspezifischen Unterschied, gibt es auch deutliche Unterschiede darin, wie weibliche und männliche lokale politische Eliten Politik vor Ort wahrnehmen und gestalten wollen. Die Einstellungen und Verhaltensweisen von Frauen und Männern in der Lokalpolitik variieren im internationalen Vergleich (Egner 2013b; Hansen und Goenaga 2019), wie auch zwischen den deutschen Bundesländern (Egner et al. 2013a; Kersting und Schneider 2016). Aber auch innerhalb der Bundesländer zeigen sich deutliche Unterschiede zwischen weiblichen und männlichen Kommunalpolitiker*innen, wobei eine Vielzahl weiterer, potenzieller erklärender Kontextvariablen wie das kommunale politische System oder sozioökonomische und kulturelle Faktoren konstant sind (Tausendpfund und Vetter 2017; Wagschal et al. 2019, S. 28). 
Über die letzten Jahre hat sich immer stärker verfestigt, dass die Einstellungen und Verhaltensweisen von lokalen politischen Eliten vor allem über deren individuelle Parteizugehörigkeit, Zugehörigkeit zu einer freien Wählergemeinschaft oder soziostrukturellen Faktoren auf der Individualebene variieren. Neuere Studien heben aber zunehmend die Bedeutung strategischer Erwägungen hervor, die aus dem interfraktionellen Rollenverständnis der Räte resultieren (Debus Gross 2016; Kersting Schneider 2016; Gross 2017) und hier zuletzt auch für den Bereich lokaler Beteiligungsverfahren im eher stark konsensdemokratischen Baden-Württemberg als signifikant getestet wurden (Ruf et al. 2019; Wagschal et al. 2019).

Obwohl in vielen Studien geschlechtsspezifische Unterschiede zwischen Ratsmitgliedern festgestellt werden konnten, wurden diese weder bei den klassischen Faktoren noch bei den neueren Überlegungen zu strategischen Erwägungen vertiefend betrachtet. Dabei sind die Bedeutung und die Auswirkung von Machtungleichgewichten, männlich dominierten Institutionen und geschlechtsspezifischen Hürden auf die Einstellungen und Verhaltensweisen von Politiker*innen hinreichend belegt (Kittilson 2006; Childs und Krook 2009; Campbell und Childs 2015; Funk und Gathmann 2015; Erzeel und Celis 2016; Barnes und Cassese 2017; Hansen und Goenaga 2019; Kim 2019). So gibt es zwischen den strategischen Überlegungen aus der Erforschung kommunalpolitischer Eliten und dem Ansatz, ein grundlegendes Machtungleichgewicht zwischen den Geschlechtern und innerhalb der Institutionen anzunehmen, durchaus Anknüpfungspunkte.

Zentrales Anliegen dieses Beitrages ist es, Unterschiede in den Einstellungen von weiblichen und männlichen Ratsmitgliedern zu bestimmten Formen der lokalen politischen Beteiligung mit einem geschlechtsspezifischen institutionalistischen Ansatz darzustellen und erste Erklärungsmöglichkeiten zu geben. Dabei wird ein Schwerpunkt auf (a) deren Parteizugehörigkeit und (b) ihren und strategischen Erwägungen gelegt. Auch wenn zu erwarten ist, dass diese Effekte im eher konsensdemokratischen Baden-Württemberg moderater ausfallen. Trotzdem, Ratsmitglieder in Baden-Württemberg haben eine zentrale Funktion als Gatekeeper, gerade beim Thema lokaler Beteiligungsmöglichkeiten. In ihrer Rolle stehen sie aber auch in einem starken Spannungsverhältnis zwischen Fraktionsinteressen, Machterhalt und Responsivität gegenüber den Bürger*innen (Copus 2010, S. 571; Ruf et al. 2019, S. 284). Darüber hinaus gibt es nicht nur signifikante Unterschiede in der Bewertung unterschiedlicher Beteiligungsmöglichkeiten auf lokaler Ebene durch Ratsmitglieder, sondern ihre Einstellungen variieren in einer Reihe von lokalen politischen Themen. Geschlechtsspezifische Unterschiede wurden hier allerdings nur rudimentär ausgeleuchtet, obwohl bereits in anderen Länderkontexten und auf anderen politischen Ebenen signifikante Unterschiede ausgemacht werden konnten und damit theoretische Annahmen über Machtungleichgewichte zwischen Männern und Frauen bestätigt wurden. Die beiden zentralen Forschungsfragen lauten daher:

1. Inwiefern haben weibliche Gemeinderatsmitglieder im Vergleich zu ihren männlichen Kollegen andere Präferenzen in Bezug auf repräsentative, partizipative und direktdemokratische Verfahren der lokalen Beteiligung? 
2. Können geschlechtsspezifische Ansätze aus der Parteienforschung und geschlechtsspezifische strategische Überlegungen (wie die Position der Befragten im Rat) ihren Teil zur Präferenzsetzung der Geschlechter erklären?

Mehrheitlich wird in den bisher geleisteten Studien ein Dualismus von zwei sich gegenüberstehenden Beteiligungsidealen aufgemacht (z. B. Karlsson 2012; Mckenna 2012; Egner et al. 2013a; Kersting und Schneider 2016). Dies wird in dieser Studie jedoch aufgebrochen, da vermutet werden kann, dass unterschiedliche Verständnisse von Ratsmitgliedern sonst nicht immer adäquat abgedeckt werden können. ${ }^{1}$ Lediglich Gabriel und Kersting (2014) beziehen sich gleichzeitig auf partizipative, deliberative und repräsentative Beteiligungsverfahren. Dieser Beitrag bietet auch weitere starke Ergänzungen zur Forschungsliteratur. So erfolgt die empirische Analyse mittels einer multinomial fractional logit (Papke und Wooldridge 1996; Mullahy 2010) eines 2017 und 2018 erhobenen Datensatzes mit 1638 Befragten Gemeinderatsmitgliedern aus Baden-Württemberg (480 weiblich, 1158 männlich). ${ }^{2}$ Damit ist der Datensatz einer der umfassendsten zu einem Bundesland und lässt auch Rückschlüsse auf die gesamte Breite der Gemeindegrößenklassen zu. Ein Großteil der Studien kam hier sonst immer schnell an ihre Grenzen. Als abhängige Variable dient die Einstellung der Befragten zu unterschiedlichen Formen der lokalen politischen Beteiligung. Dabei wird erhoben, wie die Politiker*innen zu repräsentativen (wie allgemein bindenden Personenwahlen), partizipativen (wie beratende Einwohner-

\footnotetext{
1 Die baden-württembergische Kommunalordnung lässt drei unterschiedliche Beteiligungsformen mit verschiedenen Verfahren zu, über die Bürger*innen an politischen Entscheidungen bindend oder beratend teilhaben können (Ruf et al. 2019, S. 275-276): a) Klassische repräsentative Beteiligungsangebote, in denen in Personenwahlen Gemeinderäte und (Ober-) Bürgermeister*innen in allgemeinen, bindenden Wahlen bestimmt werden. Die Bürger*innen können hier Präferenzen setzen und bei einer Wahl ihre Vertreter*in bestimmen (Heinelt 2013c, S. 105). b) Partizipative Verfahren, in denen die Bürger*innen in Dialogform beratend hinzugezogen werden könen. Formalisierte Verfahren, wie die in der Gemeindeordnung verankerte Bürgerfragestunde, Bürgeranhörung, Einwohnerversammlung oder Bürgerkonsultation. Nicht-formalisierte Verfahren, die nicht explizit in der Gemeindeordnung verankert, in ihrem beratenden Charakter den Verfahren aus der Gemeindeordnung aber ähnlich sind. Dies können Bürgerforen, Mediationsrunden, Bürgersprechstunden, die Beratung durch Beiräte aber auch explorative Bürgerbefragungen sein. c) Direktdemokratische Verfahren, die eine Entscheidung zu einem bestimmten Sachverhalt zulassen. So können dies formale Verfahren mit Entscheidungsrecht der Bürger sein, nämlich durch Rats- oder Bürgerbegehren initiierte Bürgerentscheide oder auch bürgerinitiierte, konsultative Einwohneranträge.

2 Die Verteilung der Sitzanzahl in den Gemeinderäten werden in Baden-Württemberg nicht für die einzelnen Gemeindegrößeklassen oder Gemeinden zentral erfasst. Deshalb fand eine Gewichtung nach Bevölkerungsgröße statt, um systematische Fehler bei der Beantwortung zu verhindern. Für Gemeinden zwischen 5000 und 49.999 Einwohner wurde eine Stichprobe von 100 Gemeinden gezogen. Während für Gemeinden über 50.000 Einwohner (22 Gemeinden) sowie für Gemeinden unter 5000 Einwohner (589 Gemeinden) eine Vollerhebung stattfand. Somit stehen die drei Größenklassen in einem guten proportionalen Verhältnis zueinander Insgesamt befinden sich in diesem Datensatz 2022 Befragte, die den Fragebogen bis zum Ende ausgefüllt haben. Für diese Analyse reduziert sich die Zahl in Auswertung aber auf 1638 Befragte, da einige Fragen zum Teil nicht vollständig beantwortet wurden. Die Verteilung der Befragten über die drei Gemeindegrößenklassen nach der Stichprobe und Erhebung sieht wie folgt aus: Für Gemeinden unter 5000 Einwohner*innen: 500 Befragte. Für Gemeinden zwischen 5000 und 49.999 Einwohner*innen: 1059 Befragte (720 Männer und 339 Frauen). Über 50.000 Einwohner: 463 Befragte (283 Männer und 180 Frauen). Gemessen an der Bevölkerungsgröße sind Gemeinden unter 5000 Einwohner sogar leicht überrepräsentiert (2016: 1,573 Mio Einwohner*innen in Gemeinden unter 5000 und 10,775 Mio Einwohner*innen insgesamt).
} 
versammlungen) und direktdemokratischen (wie bindende Bürgerentscheide oder beratende Einwohneranträge) Verfahren im Verhältnis zueinander stehen. Die Einstellung von politischen Eliten in solch einem lokalen Setting zu untersuchen, in dem die meisten der Kontextfaktoren konstant oder annähernd konstant gehalten werden können, verspricht zusätzliche Erkenntnisse über ihr Denken und Handeln (Kenny und Verge 2016; King et al. 1994; Snyder 2001). Daneben hängt der Erfolg von Bürgerbeteiligungsverfahren zu großen Teilen vom Willen der beteiligten Akteur*innen ab (Lowndes et al. 2006; Font und Galais 2011; Vetter 2008, S. 18-19) und von einer, wenn auch langsam, wachsenden Zahl von Frauen in politischen Ämtern.

Um die beiden zentralen Fragen zu beantworten, gliedert sich der Beitrag wie folgt: In einem zweiten Schritt wird der Forschungsbedarf weiter herausgearbeitet. Im dritten Abschnitt werden daraus anknüpfend Erklärungsfaktoren für die empirische Untersuchung abgeleitet. Nachdem im vierten Abschnitt das Forschungsdesign näher erläutert wird, folgt im fünften Abschnitt die abschließende statistische Auswertung. Im Anschluss folgt das Fazit.

\section{Forschungsstand - Gender Gap in der Literatur}

\subsection{Klassische Erklärungsfaktoren für die Einstellung lokaler politischer Eliten}

Die Zahl bisheriger Studien zur Einstellung von lokalen politischen Eliten wie Gemeinderatsmitgliedern und Bürgermeister*innen zu den unterschiedlichen Formen von Bürgerbeteiligung ist recht übersichtlich. Wie in der Literatur zur allgemeinen Einstellungsforschung lokaler politischer Eliten konzentrieren sich die Autor*innen auch hier auf eine Kombination von individuellen und kontextuellen Erklärungsfaktoren und bestätigen grundsätzlich vor allem die Kombination aus individuellen Faktoren wie der Parteizugehörigkeit, aber auch sozialstrukturellen und zuletzt auch stärker mandatsbezogene Faktoren als einflussreich (siehe zum Beispiel Karlsson 2012; Mckenna 2012; Heinelt 2013c; Egner et al. 2013b; Gabriel und Kersting 2014; Kersting und Schneider 2016; Vetter 2017; Ruf et al. 2019; Wagschal et al. 2019). ${ }^{3}$

Auch die Parteiendifferenzthese zeigt sich in Kombination mit anderen Faktoren immer wieder als einflussreich, was deren Bedeutung und die Bedeutung der Links-Rechts-Verortung von Parteien auf der kommunalen Ebene unterstreicht (zum Beispiel: Bräuninger und Debus 2012; Stecker 2017). Im Vergleich zu anderen kommunalpolitischen Systemen in Deutschland wird der Parteizugehörigkeit auf lokaler Ebene in Baden-Württemberg mehrheitlich aber eine deutlich geringere Rolle zugeschrieben. So gehen Holtkamp et al. (2015) davon aus, dass die konkordanzdemokratischen Merkmale in Baden-Württemberg zu einer geringeren Parteipolitisierung

\footnotetext{
3 Einschränkend muss aber festgehalten werden, dass sich die meisten Studien darauf konzentrieren, welche Faktoren als einflussreich im Sinne von Signifikanz getestet werden können und weniger auf die zu erklärende Gesamtvarianz. Der erklärte Gesamtzusammenhang ist dabei nur schwach, teilweise auch sehr schwach (Kersting und Schneider 2016, S. 327-330; Vetter 2017, S. 439).
} 
führen und bestimmte Personen wie einzelne Kandidat*innen und die Bürgermeister*innen eine besondere und starke Rolle spielen. Was in verschiedenen Arbeiten grundsätzlich auch bestätigt wird. Einschränkend nimmt Holtkamp aber auch an, dass abseits der skizierten Extremtypen eher Gemeinsamkeiten als Unterschiede zwischen den beiden kommunalen Entscheidungsstrukturen bestehen (Holtkamp 2006). Folglich sollte auch vielmehr von einem weniger stark parteipolitisierten System ausgegangen werden. Als ursächlich für die Entscheidungsstrukturen sieht Holtkamp für Baden-Württemberg den niedrigeren Organisationsgrad der Parteien, das Kommunalrecht sowie die kleineren Gemeindegrößen. Oft lassen sich fraktionsübergreifende Anträge und Vorhaben beobachten, die auch über Fraktionsgrenzen hinweg Bestand haben (ebd.). Holtkamp et al. (2015) nehmen weiter an, dass aufgrund des geringen Handlungsspielraumes auf kommunaler Ebene die inhaltlichen Differenzen zwischen den Parteien kaum zu einem unterscheidbaren Policy-Output führen. Ihnen also in der alltäglichen kommunalpolitischen Arbeit eine weniger starke Rolle zukommt. Studien wie von Wagschal et al. (2019) und Ruf et al. (2019) zeigen aber, dass der Einfluss der Parteiendifferenz auch in Baden-Württemberg - gerade bei der Einstellung von Ratsmitgliedern und Bürgermeister*innen zu unterschiedlichen Formen der Bürgerbeteiligung - nicht unterschätzt werden darf. Dies konnten sie auch unabhängig von der Gemeindegröße feststellen, gilt somit nicht nur für große Kommunen. Wie sehr Parteienunterschiede einen Teil der Varianz erklären können und dabei die klassische Links-Rechts-Verortung der Parteien greift, zeigen auch Heinelt (2013c, S. 120) sowie Kersting und Schneider (2016, S. 332) für unterschiedliche Formen der lokalen politischen Beteiligung in deutschen Kommunen, darunter auch baden-württembergische. So bewerten Politiker*innen der Grünen, der Linken und der SPD direktdemokratische und partizipative Beteiligungsmöglichkeiten grundsätzlich besser, als ihre Kolleg*innen von der CDU, CSU und FDP. Ein weiterer zentraler Faktor der kommunalpolitischen Parteipolitisierung unterstreicht die Bedeutung der Parteien: So verlaufen mit steigender Einwohnerzahl die Parteiensysteme immer stärker entlang der bekannten parteiideologischen Linien (Mielke und Eith 1994; Nyhuis 2016, 2017; Gross und Jankowski 2018). Allerdings konnte Uwe Wagschal et al. (2019) und Florian Ruf et al. (2019, S. 288) feststellen, dass die sonst für die kommunale Ebene so prägende Gemeindegröße (Tausendpfund und Vetter 2017, S. 4) zumindest für die Einstellung von Ratsmitgliedern und Bürgermeister*innen in Baden-Württemberg zu verschiedenen Formen lokaler Beteiligungsmöglichkeiten keinen signifikanten und wenn, nur einen sehr kleinen Einfluss hat. Ein durchaus ungewöhnlicher Befund für die kommunale Ebene, auch weil Kersting und Schneider (2016, S. 320, 332) in ihrer Studie einen stärkeren Effekt feststellen konnten, allerdings nur für 587 Befragte aus 27 verschiedenen Kommunen und für einen Teil der deutschen Bundesländer. Insgesamt ist aber zu erwarten, dass gerade Parteieneffekte im kommunalpolitischen Systems Baden-Württemberg schwächer ausfallen als in eher mehrheitsdemokratischen Systemen anderer Bundesländer, gerade mit der stärkeren Rolle freier Wählergemeinschaften. Diese Studie blickt aber auch im Besonderen auf die klassischen Parteien, auch wenn freie Wählergemeinschaften und Sonstige Listenvereinigungen als Kontrollvariable inkludiert sind. 
Zusätzlich führen ein geringerer Organisationsgrad der Parteien sowie die im Vergleich hohe Mandatszahl im Verhältnis zur Bevölkerungszahl dazu, dass es den etablierten Parteien schwerer fällt in Baden-Württemberg ihre Wahllisten zu füllen (Holtkamp 2006, S. 649). Dies ist laut Holtkamp auch eine Ursache für die zunehmende Rekrutierung parteiloser Kandidat*innen. Auch Wehling betont, dass „[g]ewählt wird, wer in der Gemeinde etwas ist, etwas gilt und wer geschätzt wird“ (Kost und Wehling 2010, S. 29). Als wichtigstes Kriterium für den Wahlerfolg sieht er eine Mitgliedschaft oder eine Funktion in einem Verein. Darüber hinaus betont er die hohe „Kontinuität in der Zusammensetzung der Gemeinderäte“ (ebd.: 30). Mit Blick auf den geringen Frauenanteil in den Gemeinden nennt Wehling vor allem zwei Gründe. So seien Frauen deutlich weniger in bedeutenden Berufen und Vereinen vertreten und zuletzt seien traditionelle Rollenverteilungen zwischen Mann und Frau immer noch sehr stark ausgeprägt (ebd.). In dieser Frage konnte zuletzt Florian Ruf (2019) ausdifferenzieren, dass aber auch das Vorhandensein oder die Abwesenheit von parteiinternen Fördermaßnahmen, sowie die Frage weiblicher Gatekeeper im Rekrutierungsprozess einen starken Einfluss auf die Nominierung von Frauen auf kommunale Wahllisten in Baden-Württemberg haben. Somit also auch Variablen, die auch auf kommunaler Ebene innerhalb und zwischen den Parteien (oder Wahllisten) variieren und wiederum für einen gewissen Grad an Parteipolitisierung in diesem Themenfeld sprechen, wenn auch vermutlich in einem geringeren Umfang als in mehrheitsdemokratischen Systemen.

\subsection{Bedeutung strategischer Erklärungsfaktoren}

Wenn politische Akteur*innen ihre Präferenzen umsetzen wollen, müssen sie strategisch denken. Wie sehr diese sich strategisches Verhalten zu Nutze machen zeigt Kenneth A. Shepsle (2010, S. 159) und führt aus, dass für Strategie hier eine gewisse Vorausschau in der Erwartung eines Eintreffens eines Ereignisses bedeutet. Dafür werden Präferenzen gesetzt und es wird versucht mögliche Wege vorzuzeichnen, um die priorisierte Präferenz dann durchzusetzen (Shepsle 2010, S. 183-184). Auch kommunalpolitische Akteur*innen sind bis zu einem gewissen Grad rational handelnde Akteur*innen mit Präferenzen, die sie umsetzen möchten.

Wie sehr strategische Erwägungen für die Erklärung lokaler Politik in Deutschland an Bedeutung hinzugewonnen hat, konnten Debus und Gross (2016) anhand von Koalitionsbildungen auf lokaler Ebene darlegen. So versuchen Ratsmitglieder und Bürgermeister*innen, ihren Nutzen zu maximieren, in dem sie auf Basis strategischer Erwägungen entsprechend Koalitionen eingehen. Diese Erwägungen kann auch Gross (2017) für den selbstwahrgenommenen Machteinfluss lokaler politischer Eliten in 27 hessischen Kommunen bestätigen. So hängt dessen Stärke vor allem davon ab, ob sich die Befragten einer kommunalpolitischen Mehrheit wie der Partei des Bürgermeisters oder der Mehrheitsfraktion im Rat zurechnen, aber auch ihre politische Erfahrung und ihre Position im lokalen Machtsystem haben einen Einfluss (Gross 2017, S. 144). Zwar sind diese Effekte vor allem in mehrheitsdemokratischen kommunalpolitischen Systemen wie Nordrhein-Westfalen und Hessen stark, die Frage ob Mehrheit oder Minderheit im Rat spielt aber beim Thema der kommunalen Beteiligungsmöglichkeiten auch in Baden-Württemberg eine Rolle. Hier 
konnten zuerst Kersting und Schneider (2016) einen negativen Effekt auf die Einstellung von Ratsmitgliedern zu Bürgerbeteiligung in den Bundesländern feststellen und unterstreichen damit die Bedeutung der Zugehörigkeit zur Mehrheitsfraktion und die Unterscheidung zu den Minderheitsfraktionen. Gleiches konnten Wagschal et al. (2019) umfassend für Bürgermeister*innen in Baden-Württemberg bestätigen, unabhängig von der Gemeindegröße. Ähnliche strategische Überlegungen von Ratsmitgliedern in Baden-Württemberg treten auch in der Arbeit von Ruf et al. (2019, S. 289) zutage: Ratsmitglieder, die mit der Durchführung von mehr Beteiligungsverfahren in ihrer Kommune einen Machtverlust des Rates befürchten, befürworten vor allem klassische repräsentative Beteiligungsverfahren, unabhängig von der Gemeindegröße.

Zumindest für das Feld der kommunalen Beteiligungsmöglichkeiten gibt es in Baden-Württemberg auch doch oft ein Denken und Handeln der Ratsmitglieder innerhalb von Mehrheit und Minderheit im Rat. Mögliche Erklärungen könnte die lange Tradition und Bedeutung von partizipativen und direktdemokratischen Verfahren in Baden-Württemberg sein oder die vielleicht etwas stärkere Polarisierung und Mobilisierung aufgrund der Themen, die über partizipative oder direktdemokratische Verfahren behandelt werden. So stehen solche Verfahren und die Positionen der Ratsmitglieder dazu in einem deutlich stärkeren Öffentlichkeitsinteresse, als die alltägliche kommunalpolitische Arbeit. Einen grundsätzlicheren Befund liefern Debus et al. (2012): Auch in den baden-württembergischen Kommunen geht es bei bestimmten Policies um Verteilungsfragen, die entlang parteipolitsicher Linien entschieden werden, wie von den Autoren in der untersuchten Frage zu Örtlichkeiten und Gebührenhöhe bei der Schließung gleichgeschlechtlicher Lebenspartnerschaften gezeigt wird.

\subsection{Machtungleichgewichte und geschlechtsspezifische Erklärungsfaktoren}

Macht spielt nicht nur in strategischen Überlegungen eine sehr wichtige Rolle, sondern wird auch in Form von Machtungleichgewichten zwischen Geschlechtern als Erklärungsansatz in gendersensiblen Forschungsansätzen verwendet. Hier verspricht ein geschlechtsspezifischer institutionalistischer Ansatz wichtige Erkenntnisse über das Zusammenspiel und die Funktionsweise von politischen Institutionen und das Verhalten seiner Akteur*innen (Kenny 2007). In diesem Zusammenhang und in Anlehnung an die Ausführungen von Shepsle (2010), wird Macht neben Strategie als weiteres nötiges Mittel zur Durchsetzung der eigenen oder der Gruppenpräferenzen definiert. Dies kann sowohl Macht als Gatekeeping-Autorität (gatekeeping authority) oder auch Macht im Sinne eines Vorschlagsrecht (proposal power) sein.

Aus der Sicht eines gendersensiblen institutionalistischen Forschungsansatzes sind politische Institutionen wie Parteien, Listenvereinigungen und Parlamente keine geschlechtsneutralen Institutionen und Akteure, die rein rational handeln (Hoecker 1995; Lovenduski 2005; Kittilson 2006; Celis et al. 2016; Childs und Kittilson 2016). Vielmehr ist Macht als strategisches Mittel zur Durchsetzung von Präferenzen zwischen den Geschlechtern überwiegend ungleich verteilt. Ein Blick nach Baden-Württemberg zeigt, dass dort Ratsfrauen auch heute noch deutlich in der Unterzahl (Statistik BW: 26,8\%) und auch nur rund $8 \%$ der Ratsvorsitzenden stel- 
len (Schwarzwälder Bote 2020). Auch der hohe Grad an Dezentralisierung bei der Nominierung von Kandidat*innen (Hazan und Rahat 2010), dem Widerstand gegen mehr Zentralisierung bei der Nominierung, sowie fehlende Fördermaßnahmen, der oft hohe Anteil an freien Listenvereinigungen ohne übergeordnete Strukturen, sowie von Männern dominierte Gatekeepingpositionen der Parteien- und Listenvereinigungen deuten darüber hinaus auf stark männlich dominierte Strukturen hin, die sich wiederum stark auf den geringen Anteil an Frauen auf den kommunalen Wahllisten auswirken (Ruf 2019).

Daraus resultierende Machtungleichgewichte in politischen Institutionen haben unterschiedliche Auswirkungen auf die Verhaltensweisen und Einstellungen der Politiker*innen. So begünstigen gesellschaftliche Normen, formelle und informelle Regeln in Parteien und Parlamenten bestehende Akteure in einflussreichen Positionen (Norris 2004; Lovenduski 2005; Verge und Claveria 2016), die für gewöhnlich sehr stark von Männern dominiert werden. ${ }^{4}$ Frauen befinden sich daher institutionell oft in informellen Minderheitenpositionen, da alleine schon numerisch zum Beispiel Gatekeeping-Autorität und Vorschlagsrecht innerhalb politischer Institutionen und zwischen den Geschlechtern nicht gleich verteilt sind. Somit haben es Ratsfrauen deutlich schwieriger, ihre Präferenzen als Individuum oder Gruppe im Rat genauso gut durchzusetzen wie ihre männlichen Kollegen. Dieses institutionelle Machtungleichgewicht kann zu unterschiedlichen (geschlechtsspezifischen) Einstellungen führen. Unterschiede in den Einstellungen und Verhaltensweisen von lateinamerikanischen Abgeordneten stellt so auch Schwindt-Bayer (2006) fest und erklärt dies mit Marginalisierungseffekten parlamentarischer Institutionen, die sie in ihrer Studie auch auf parlamentarische Institutionen in Westeuropa übertragen kann. Nun können diese Ergebnisse nicht eins zu eins auf das kommunalpolitische System Baden-Württembergs übertragen werden. Grundsätzlich zeigt aber eine Reihe von Studien aus den unterschiedlichsten Kontexten, dass bei einer Vielzahl von Themen und in unterschiedlichen politischen Systemen ein substanzieller Gender Gap in den Einstellungen zwischen Männern und Frauen auch aufgrund von Machtungleichgewichten existiert (Kittilson 2006; Schwindt-Bayer 2006; Childs und Krook 2009; Wängnerud 2009; Childs 2010; Brunsbach 2011; Celis und Childs 2012; Chaney 2014; Funk und Gathmann 2015; Erzeel und Celis 2016; Barnes und Cassese 2017; Blome und Fuchs 2017; Hansen und Goenaga 2019). ${ }^{5}$ Wie stark oder schwach dieser Effekt ausfällt, hängt natürlich auch von der Parteizugehörigkeit, sozioökonomischen Rahmenbedingungen wie Alter, Bildungsgrad sowie der Links-RechtsPosition von Parteien und der ideologischen Positionierung von Frauen und Männern ab. Machtungleichgewichte oder marginalisierende Effekte konnten aber bisher

\footnotetext{
${ }^{4}$ Bekannt ist der aus der Erforschung politischer Rekrutierungsprozesse problematisierte Effekt, dass parteipolitische Schlüsselpersonen neues Personal rekrutieren, die ihrem Verhalten, Einstellungen, sozialem Milieu oder eben auch Geschlecht sehr ähnlich sind oder stereotypen Erwartungen an die Aufgabenerfüllung am ehesten Entsprechen (siehe z. B. Pruysers und Blais 2017). Dieser Vorgang wird auch häufig als „Selbstrekrutierung“ bezeichnet.

${ }^{5}$ Dem Forschungsinteresse liegt meist die Annahme zugrunde, dass eine höhere deskriptive Repräsentation von Frauen eine stärkere substanzielle Repräsentation von Frauen zur Folge hat (Phillips 1995). Die meisten dieser Arbeiten beschäftigen sich zudem damit, ob und unter welchen Bedingungen Frauen die Interessen von Frauen vertreten.
} 
in allen politischen Systemen und auf den unterschiedlichsten Ebenen beobachtet werden. Klassische Faktoren der Einstellungsforschung bilden dabei den Rahmen für die Richtung, die Stärke und die Signifikanz geschlechtsspezifischer Effekte. Mit Blick auf das eher konsensdemokratische System Baden-Württembergs ist demnach für die Räte ein eher moderater Effekt zu erwarten.

Gerade in der Erforschung der substanziellen Repräsentation von Frauen wurden parteipolitische und ideologische Erklärungsansätze stark ausdifferenziert (siehe zum Beispiel Erzeel und Celis 2016). Grundsätzlich wird in der Erklärung ein Unterschied zwischen linken und rechten Parteien, aber auch aufgrund der ideologischen Positionierung von politischen Eliten als einflussreich angesehen (NORRIS et al. 1992; Kittilson 2006; Erzeel und Celis 2016). Mit Blick auf die Einstellungen zu unterschiedlichen Formen der lokalen Beteiligung von Ratsmitgliedern in Deutschland und anderen europäischen Ländern stellt Heinelt (2013a, S. 88, 2013b, S. 651, 2013c, S. 120) in mehreren Arbeiten fest, dass Frauen partizipative Beteiligungsformen stärker, repräsentative Beteiligungsformen hingegen schwächer befürworten als ihre männlichen Kollegen. Er führt diesen Unterschied aber nicht weiter aus. Zumindest für die Bewertung partizipativer Beteiligungsformen können dies auch Ruf et al. (2019, S. 287) für baden-württembergische Ratsmitglieder bestätigen. Auch die Arbeit von Denters und Klok (2013) bestätigt diesen Effekt und geht in ihrer Erklärung auf die unterschiedlichen Effekte ein, die verschiedene politische Systeme und Institutionen auf die Bewertung von Beteiligungsformen und auf die Responsivität der Ratsmitglieder haben können und sehen einen positiven Zusammenhang in der Bewertung weiblicher Ratsmitglieder gegenüber partizipativen Beteiligungsmöglichkeiten (Denters und Klok 2013, S. 671). Hingegen kann Vetter (2017, S. 433-434) in 28 hessischen Gemeinden keine Belege dafür finden, dass die männlich dominierte Institution des Gemeinderats dazu führt, dass Frauen Bürgerbeteiligung besser bewerten als ihre Kollegen. Dies bestätigen in einer ähnlichen Arbeit auch Kersting und Schneider (2016, S. 327). Leininger (2015, S. 22) und Bengtsson und Mattila (2009, S. 1042) zeigen in zwei europaweiten Studien, dass Frauen direktdemokratische Instrumente besser bewerten als Männer. Wie sehr aber politische Institutionen die Einstellungen und Verhaltensweisen von Männern und Frauen beeinflussen können, konnten Hansen und Goenaga (2019) anhand des European Social Survey Datensatzes aufzeigen. Ihnen zufolge bewerten Frauen klassische repräsentative Institutionen und ihre Verfahren als weniger bedeutsam als Männer und bevorzugen dafür partizipative und direktdemokratische Verfahren und Verhaltensweisen. Als Erklärung für diesen Unterschied nennen die Autor*innen die Zuschreibungen sozialer Gruppen und deren Erwartungshaltung gegenüber dem demokratischen System und wie sehr darin ein demokratisches Instrument diese soziale Gruppe in ihrer Teilhabe fördert oder behindert, in diesem Fall also geschlechtsspezifische Ungleichheiten reproduziert (Hansen und Goenaga 2019, S. 5).

Insgesamt fehlt es an Studien, die umfassend geschlechtsspezifische Einstellungen von politischen Eliten in Deutschland untersuchen, gerade auf kommunaler Ebene. Unabhängig von den politischen Systemen wurden aber im überwiegenden Teil international- oder national-vergleichender Studien, geschlechtsspezifische Effekte festgehalten. Für die kommunale Ebene in Deutschland wird für diese Arbeit 
deshalb auch auf Erkenntnisse aus diesen Arbeiten zurückgegriffen. Auch wenn diese nicht eins zu eins auf das kommunalpolitische System in Baden-Württemberg übertragen werden können, liefern sie doch wichtige Hinweise für die geschlechtsspezifische Funktionsweise politischer Institutionen und das Handeln und Denken ihrer Akteur*innen. Hier deutet vieles darauf hin, dass nicht das ob, sondern vielmehr Stärke und Varianz variieren. Zusätzlich liefert die Arbeit von Florian Ruf et al. (2019, S. 287) einen ersten Beleg dafür, dass im Bereich der Bürgerbeteiligung in Baden-Württemberg die Einstellung der Ratsmitglieder auch nach Geschlecht variiert.

\section{Hypothesen}

Machtungleichgewichte innerhalb politischer Institutionen zwischen Männern und Frauen begünstigen Männer in ihrer dominierenden Position und ihrem Einfluss auch auf kommunaler Ebene. In den baden-württembergischen Gemeinderäten sind Frauen deutlich weniger vertreten als Männer (Ruf 2019) und an der Ratsspitze befindet sich nur in seltenen Fällen eine Frau (Wagschal et al. 2019, S. 19). Diskriminierende Effekte in parlamentarischen und parteipolitischen Institutionen lassen sich bei der Rekrutierung und Auswahl von geeigneten Kandidat*innen feststellen, in deren Prozess vor allem männerdominierte Netzwerke über zukünftige Kandidierende entscheiden. In diesen Netzwerken wird ein Empowerment von Frauen zumeist skeptisch betrachtet und entsprechend werden eher zögerlich auftretende Frauen zugleich seltener rekrutiert (Butler und Preece 2016; Lawless und Fox 2005; Pruysers und Blais 2017). Gleichzeitig schreiben wichtige Gatekeeper aufgrund stereotyper Auswahlkriterien die für eine Kandidatur nötigen Kompetenzen und Fähigkeiten häufiger Männern zu, die entsprechend dann auch häufiger ausgewählt werden (Ruf 2019). Ressourcenbasierte Ansätze führen darüber hinaus ins Feld, dass Frauen häufiger für das klassische politische Engagement wichtigen Ressourcen, wie Zeit und Geld, fehlen (Verge und Claveria 2016). Gleichzeitig sind Abläufe und Debatten in Parlamenten, Ausschüssen und Parteien in ihrer Arbeit oft diskriminierend gegenüber Frauen und bevorteilen Männer (Baekgaard und Kjaer 2012; Bäck et al. 2014; Krook und Norris 2014). All diese Effekte führen dazu, dass geschlechtsspezifische Ungleichheiten durch diese Institutionen reproduziert werden können und Frauen in diesen Institutionen schlechtere Teilhabechancen haben als Männer (Hansen und Goenaga 2019, S. 5). Diese Erfahrungen könnten sich auch auf die grundsätzliche Einstellung von Frauen auf die Gestaltung von Politik auf lokaler Ebene auswirken. Demnach könnten Gemeinderät*innen auf lokaler Ebene in Baden-Württemberg gegenüber dem klassisch repräsentativen System und seinen Verfahren eine skeptischere Erwartungshaltung haben als ihre männlichen Kollegen, da diese sie in vielen Punkten aus benachteiligen. Dies führt zu folgender Hypothese:

H1a: Weibliche Ratsmitglieder bewerten repräsentative Beteiligungsverfahren schlechter als ihre männlichen Kollegen.

Hingegen sind für Beteiligungsformen, die außerhalb klassischer repräsentativer Beteiligungsverfahren liegen, andere Effekte zu erwarten. Entscheidungen über 
partizipative und direktdemokratische Beteiligungsformen umgehen bestimmte repräsentative Entscheidungsprozesse, ihre Institutionen und ihre Auswirkungen auf die Verhaltensweisen und Einstellung von Männern und Frauen, auch wenn diese natürlich nicht unabhängig von ihnen im luftleeren Raum stattfinden (Hornig 2011). Frauen legen oft andere Kriterien an eine erfolgreiche Beteiligung als Männer und sie schreiben partizipativen und direktdemokratischen Beteiligungselementen Eigenschaften zu, die ihrer Erwartungshaltung an ein mögliches Ergebnis eher entsprechen (Hansen und Goenaga 2019, S. 6). Da repräsentative Strukturen, Verfahren und Beteiligungsmöglichkeiten ihre Basis und Schwerpunkte in Parteien, Fraktionen und den Ratsgremien haben, welche Männer in der Summe eher bevorteilen und Frauen eher benachteiligen (Verge und Claveria 2016), suchen Frauen aber möglicherweise einen partizipativen oder direktdemokratischen Ausweg, um ihre Interessen besser durchzusetzen (Shepsle 2010, S. 183-184). Wie sehr direktdemokratische Referenden Geschlechtergerechtigkeit befördern können und damit im strategischen Interesse von Frauen stehen können, konnte zuletzt auch Yeong Hyun Kim (2019) feststellen. Aufgrund dieser Überlegungen wird ergänzend folgende Hypothese getestet:

H1b: Weibliche Ratsmitglieder bewerten partizipative und direktdemokratische

Beteiligungsverfahren besser als ihre männlichen Ratskollegen.

Aufbauend auf dem Machtungleichgewicht innerhalb politischer Institutionen sind auch bestimmte geschlechtsspezifische Parteieneffekte zu erwarten. Linke Parteien befürworten partizipative und direktdemokratische Beteiligungsmöglichkeiten grundsätzlich stärker als rechtsorientierte Parteien, die wiederum repräsentative Verfahren stärker befürworten (Heinelt 2013c, S. 120; Kersting und Schneider 2016, S. 327; Ruf et al. 2019, S. 284). Das spiegelt sich auch in der Programmatik der Parteien wieder. Diese klassische Annahme macht keine geschlechtsspezifischen Aussagen, obwohl für die Erklärung substanzieller Unterschiede zwischen Frauen und Männern, Parteien und die ideologische Positionierung von politischen Eliten als sehr einflussreich angesehen werden (NORRIS et al. 1992; Kittilson 2006; Erzeel und Celis 2016). Campbell und Childs (2015) konnten zudem aufzeigen, dass ideologische Unterschiede zwischen Frauen und Männern innerhalb der Conservative Party in Großbritannien erklären können, welche Policy-Präferenzen vertreten werden und, dass sich Frauen und Männer auch innerhalb der gleichen Partei stark voneinander unterscheiden können. Gemäß dem zuvor skizzierten Machtungleichgewicht in politischen Institutionen sollten Frauen andere strategische Überlegungen aufstellen und ihre Einstellungen daraufhin anpassen, da es sie sind, die aufgrund von männlich dominierten Institutionen und Strukturen in ihren Teilhabechancen eingeschränkt werden und andere Entscheidungsformate suchen (müssen), um ihre Prioritäten besser durchzusetzen (Shepsle 2010, S. 183-184). Zusätzlich könnte das Ausmaß des zu erwartenden Machtungleichgewichts auf die strategischen Überlegungen von Frauen und Männern durch ihre Parteizugehörigkeit moderiert werden. So sind große Unterschiede in den Policy-Präferenzen zwischen den Geschlechtern vor allem dort zu erwarten, wo das Machtungleichgewicht in der Partei größer ausfällt und es Frauen die Teilhabe stärker erschwert, als in anderen Parteien. Barnes und Casses (2017, S. 136) konnten für die USA aufzeigen, dass geschlechtsspe- 
zifische Unterschiede vorwiegend innerhalb der Republikanischen Partei auftreten und geschlechtsspezifische Unterschiede bei der demokratischen Partei deutlich geringere Effekte auf die Policy-Präferenzen von Frauen und Männern haben. Daraus lässt sich schließen, dass sich Frauen und Männer in ihren Präferenzen innerhalb der demokratischen Partei ähnlicher sind. Barnes und Casses (2017) vermuten deshalb auch, dass Machtungleichgewichte in der demokratischen Partei kleiner sind, als in der republikanischen Partei. Vor allem linksorientierten Parteien wird zugeschrieben, dass sie Maßnahmen ergreifen, um Geschlechterdiskriminierung in ihren Strukturen entgegenzuwirken, um damit Machtungleichgewichte zu verringern (zum Beispiel Sundström und Stockemer 2015, S. 266). Für Baden-Württemberg konnte Florian Ruf (2019, S. 20) zeigen, in welchem Ausmaß Parteien auf der Kommunal- und Landesebene daran arbeiten, diskriminierende Effekte mit Förderstrukturen auszugleichen und das hier grundlegende Unterschiede zwischen linken und rechten Parteien bestehen. Die Erkenntnisse deuten darauf hin, dass die Machtungleichgewichte in linksorientierten Parteien geringer sind oder hier zumindest stärker ausgeglichen werden und der Widerstand gegen Förderstrukturen niedriger ist, als in rechtsorientierten Parteien. Die Policy-Präferenzen von Frauen und Männern unterscheiden sich auch deshalb stärker innerhalb rechtsorientierter Parteien, da dort weniger Maßnahmen ergriffen werden, um Machtungleichgewichten entgegenzuwirken. Bei Frauen aus rechtsorientierten Parteien sollte deshalb ein viel stärkerer Anreiz darin liegen, in bestimmten Beteiligungsformen einen strategischen Ausweg zu suchen, um ihre eigenen Policy-Präferenzen durchzusetzen. Davon sollten ihre skeptischeren Einstellungen, gegenüber partizipativen und direktdemokratischen Instrumenten, aufgrund ihrer parteipolitischen Zugehörigkeit jedoch unberührt bleiben. Trotzdem: weibliche Ratsmitglieder bewerten partizipative und direktdemokratische Beteiligungsverfahren besser, als ihre männlichen Ratskollegen $(\mathrm{H} 1 \mathrm{~b})$ und sie bewerten repräsentative Beteiligungsmöglichkeiten schlechter als ihre männlichen Kollegen (H 1a). Unterschiedliche Niveaus von Machtungleichgewichten zwischen den Parteien können diesen Effekt aber zusätzlich moderieren. Ergänzend zu Hypothese 1a und 1b lautet die nächste Hypothese demnach:

H2: Weibliche Ratsmitglieder aus linksorientierten Parteien unterscheiden sich in ihrer Bewertung partizipativer und direktdemokratischer Beteiligungsmöglichkeiten weniger von ihren männlichen Partei- und Listenkolleg*innen, als dies bei Frauen und Männern rechtsorientierter Parteien der Fall ist.

Ratsmitglieder und Bürgermeister versuchen ihren Nutzen zu maximieren, in dem sie auf Basis strategischer Erwägungen entsprechend Koalitionen auf Gemeindeebene eingehen (Gross 2017). Dieser Effekt besteht auch bei der Präferenzsetzung politischer Eliten zu lokalen Beteiligungsmöglichkeiten (Kersting und Schneider 2016; Ruf et al. 2019; Wagschal et al. 2019). Auch Ratsmitglieder in Baden-Württemberg können sich auf lokaler Ebene formal in drei Rollen befinden, aus denen sie strategische Erwägungen ableiten: Der Ratsminderheit, der Ratsmehrheit oder sind fraktionslos. Ebenfalls durch die Linse des herrschenden Machtungleichgewichts und analog zu Hypothese 1a und $1 \mathrm{~b}$ lässt sich für diese Erwägungen vermuten, dass für Frauen die oben genannten Effekte weniger geltend gemacht werden können. Im Vergleich zu ihren männlichen Kollegen befinden sie sich bereits in einer 
Art Minderheitenposition, egal welche formale Zugehörigkeit sie innehaben. Der Überlegung liegt demnach zugrunde, dass Frauen zusätzliche Hindernisse überwinden müssten, bevor sie ihre Präferenzen genauso wie ihre männlichen Kollegen durchsetzen können (Shepsle 2010, S. 183-184). Folglich ist zu erwarten, dass die Zugehörigkeit zu einer der drei strategischen Rollen auf die Bewertung der Beteiligungsmöglichkeiten durch männliche Ratsmitglieder einen Einfluss hat, weniger allerdings auf die Bewertung durch weibliche Ratsmitglieder.

Strategische Überlegungen im kommunalpolitischen Systems Baden-Württemberg als Erklärungsfaktor heranzuziehen scheint auf den ersten Blick eher kontraintuitiv zu sein. Allerdings geht es hier nicht um die Frage, ob Baden-Württemberg eher konkordanz- oder konkurrenzdemokratisch, konsens- oder mehrheitsdemokratisch aufgestellt ist. Hier ist die Forschungsliteratur in ihrem Urteil sehr eindeutig in Richtung konkordanz- oder konsensdemokratisch. Vielmehr geht es um die Frage, ob Frauen im Verhältnis zu ihren männlichen Ratskollegen beim Thema Beteiligungsverfahren aus ihrer Zugehörigkeit zur Mehrheitsfraktion, zu der sie sich in der Befragung selbst zugeordnet haben, andere Schlüsse ziehen als ihre männlichen Kollegen. Denn auch wenn der Effekt der Mehrheitsfraktion vor allem in Hessen oder Nordrhein-Westfalen zum Vorschein tritt, konnten gerade auch für die kommunale Ebene in Baden-Württemberg bereits zuvor Effekte bei der Frage der Zugehörigkeit zur Mehrheit im Rat in Untersuchungen für alle Genmeindegrößeklassen festgestellt werden, die sich im Besonderen auch auf den hier vorliegenden Frageschwerpunkt lokaler Beteiligungsverfahren beziehen (Wagschal et al. 2019; Ruf et al. 2019). Es lassen sich also auch hier Effekte erwarten.

Folglich lautet die abschließende Hypothese:

H3: Auf männliche Ratsmitglieder hat ihre formale Zugehörigkeit zur Minderheits- oder Mehrheitsfraktion einen deutlich stärkeren Effekt auf die Bewertung der unterschiedlichen Beteiligungsmöglichkeiten als auf weibliche Ratsmitglieder.

\section{Daten, Operationalisierung und Analysestrategie}

Die Einstellung der Gemeinderäte zu repräsentativen, direktdemokratischen und partizipativen Beteiligungsformen bilden die drei abhängigen Variablen in dieser Arbeit. Ihre Messung erfolgte dabei so, dass die Befragten auf drei Schiebreglern insgesamt 100 Punkte verteilen konnten. Je mehr Punkte eine Beteiligungsform erhält, umso mehr wird sie vom Befragten präferiert (Wagschal et al. 2019; Ruf et al. 2019). Hinter diesem Vorgehen steht die Idee, dass es sich um drei distinkte Typen politischer Beteiligungsformen handelt, unter denen ein Nullsummenspiel vorherrscht. Bewertet ein Ratsmitglied repräsentative Verfahren mit hundert von hundert Punkten muss es in der Summe Punkte in gleicher Höhe bei den anderen beiden Beteiligungsmöglichkeiten abziehen. Die Werte gleichen sich also immer aus. So können beispielsweise Personen, die absolute Verfechter repräsentativer Beteiligungsverfahren sind, nicht gleichzeitig auch direktdemokratische oder partizipative Verfahren gutheißen. Entsprechend können die drei getrennten abhängigen Variablen, auch als 
Präferenzordnung interpretiert werden. Die Daten für diese Untersuchung stammen aus dem Forschungsprojekt zur Einstellung kommunalpolitischer Eliten zur Bürgerbeteiligung in Baden-Württemberg (Ruf et al. 2019; Wagschal et al. 2019) von 2017 und 2018 sowie aus ergänzend recherchierten Strukturdaten der Gemeinden. Einige Kennwerte, wie die Gemeindegröße oder die Anzahl effektiver Parteien, wurden über die Seite des Statistischen Landesamtes Baden-Württemberg oder den Internetauftritt der Gemeinden recherchiert. Fehlende Werte wurden durch Mittelwerte imputiert. Die Auswertung erfasst 1638 baden-württembergische Gemeinderäte aus allen Gemeindegrößenklassen. ${ }^{6}$ In der Erhebung wurde gezielt auf eine Befragung mit möglichst vielen Ratsmitgliedern aus allen Gemeindegrößen gesetzt, um möglichst umfassende Aussagen für alle Gemeindegrößen zu treffen. Dabei lag der Schwerpunkt der Erhebung nicht auf geschlechtsspezifischen Unterschieden zwischen den Ratsmitgliedern, die hier nachträglich als weitere Analysebene eingefügt wurde. Neben der überwiegend quantitativen Ausrichtung der Untersuchung wurden ergänzend in dem Forschungsprojekt für drei Kommunen auch eine qualitative Auswertung durchgeführt, die ihren Schwerpunkt auf der Kommunikation bei Beteiligungsverfahren legt (siehe Krummenauer et al. 2019).

Die beobachtenden Ausprägungen im Datensatz sind die Einstellungen der Ratsmitglieder zu repräsentativen, partizipativen und direktdemokratischen Beteiligungsmöglichkeiten. Sowohl systemische Faktoren auf der Individualebene als auch auf der lokalen und regionalen Ebene können dabei zur Erklärung beitragen. Die Variablen variieren entweder zwischen den Ratsmitgliedern (Geschlecht, Parteizugehörigkeit, Zugehörigkeit zur Mehrheitsfraktion, Amtszeit, Alter, Hochschulabschluss, drohender Machtverlust Rat/OB) oder auf der kommunalen Ebene (Wahlbeteiligung, Arbeitslosenquote, Vereinsdichte, Haushaltsverschuldung, Gemeindegröße, Anzahl effektiver Parteien). Die Hypothesen werden in unterschiedlichen Modellen getestet, jeweils für repräsentative, partizipative und direktdemokratische Beteiligungsformen

Tab. 1 Modellaufbau. (Eigene Darstellung.)

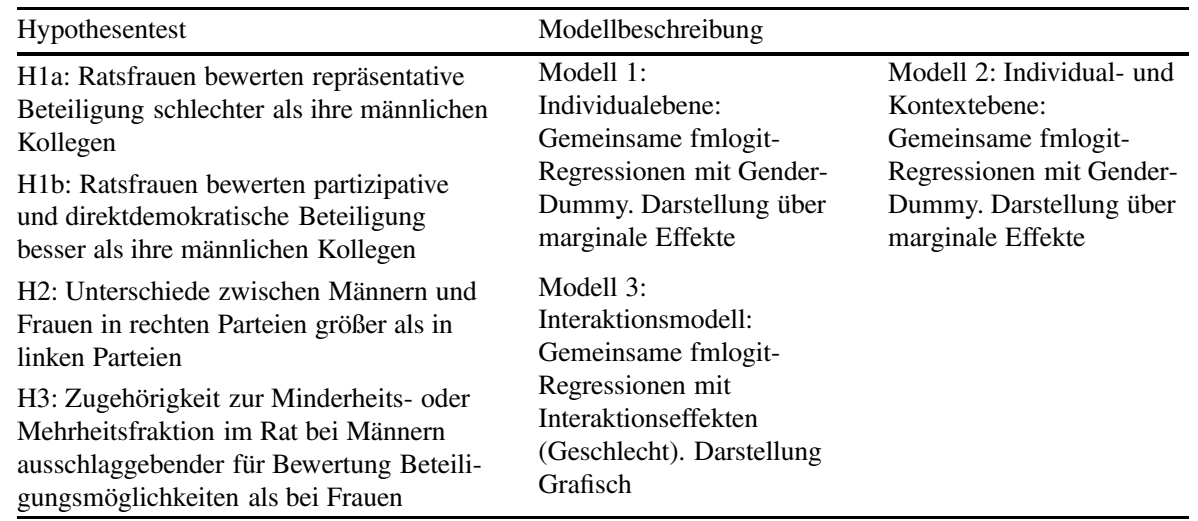

\footnotetext{
6 Die Verteilung der Befragten über die Parteien und Listen sieht wie folgt aus: CDU (N=487, Frauen=114, Männer=373), FW (N=448, Frauen=115, Männer=333), SPD (N=357, Frauen=138, Männer=219), Grünen $(\mathrm{N}=266$, Frauen=129, Männer=137), Linken $(\mathrm{N}=26$, Frauen=8, Männer=18), FDP $(\mathrm{N}=50$, Frauen=15, Männer=35), sonstige Listenvereinigungen (N=384, Frauen=103, Männer=281).
} 
(siehe Tab. 1). Da die abhängigen Variablen die Einstellung zu den Beteiligungsmöglichkeiten in Abhängigkeit zu den jeweils anderen beiden Beteiligungsmöglichkeiten abbilden, ist eine Methode notwendig, die dieses gegenseitige Verhältnis modelliert (Aitchison 2003). Dafür werden die drei abhängigen Variablen in einer fractional multinomial logit (fmlogit) (Buis 2008; Mullahy 2010) modelliert, einer multivariaten Erweiterung der fractional logit Modelle von Papke und Wooldridge (1996). Die abhängigen Variablen müssen dafür zwischen 0 und 1 liegen und in ihrer Summe stets 1 ergeben. Die Einstellung der Befragten wird dafür in ihrer relativen Ausprägung für die Modellierung operationalisiert. Für die Erfassung der abhängigen Variablen wird jeweils der proportionale Wert operationalisiert und die marginalen Effekte jeweils nach den AVs (1) repräsentativer Beteiligung, (2) direktdemokratischer Beteiligung und (3) partizipativer Beteiligung berechnet.

Innerhalb einer fractional multinomial logit ist die Darstellung der erklärten Gesamtvarianz im Sinne eines $\mathrm{R}^{2}$ oder Pseudo- $\mathrm{R}^{2}$ nicht möglich. Dies kann hier als Hinweis nur über OLS-Regressionen dargestellt werden. Ebenfalls gilt dies für die Werte zur Varianzinflation (VIF), welche aber auf keine Multikollinearität hinweisen. Ausreißer, die eine Modellschätzung zu stark beeinflussen, konnten ebenfalls nicht identifiziert werden (berechnet wurden Cook-Distanz und leverage). In einem ersten Schritt wird über eine Dummy-Variable die grundsätzliche Frage nach dem Einfluss des Geschlechts auf die Einstellung beantwortet (Hypothesentest 1a und 1b). Dabei werden zuerst die Effekte nur für die Individualebene, anschließend für die Individual- und Kontextebene berichtet. In einem dritten Schritt werden über Regressionen mit Interaktionseffekten die unterschiedlichen Effekte bei Frauen und Männern beleuchtet (Hypothesentest 2 und 3). Der Datensatz beinhaltet 480 Frauen und 1158 Männer und kommt der Verteilung in der Grundgesamtheit damit sehr nah, auch wenn Männer in einzelnen Gruppen leicht überrepräsentiert sind. Auf eine nachträgliche Gewichtung wurde aber verzichtet.

Die Operationalisierung der zentralen Variablen findet wie folgt statt: Die Parteizugehörigkeit der Ratsmitglieder wird mit Dummies je nach Parteifärbung abgebildet. Die Frage nach der Zugehörigkeit zur Mehrheit im Gemeinderat wird ebenfalls über einen Dummy operationalisiert, hier steht 1 für die Zugehörigkeit des Befragten zur Mehrheit im Gemeinderat. Das Geschlecht wurde ebenfalls über eine DummyVariable operationalisiert $(1=$ Frau $)$.

\section{Auswertung}

Zu Beginn wurde die Frage gestellt, welche Präferenzen weibliche Gemeinderatsmitglieder bei lokaler Beteiligung im Vergleich zu ihren männlichen Kollegen setzen und ob insbesondere geschlechtsspezifische Ansätze Unterschiede in der Präferenzsetzung zwischen Ratsfrauen und Ratsmännern erklären können. Dafür wurden vier unterschiedliche Hypothesen aufgestellt. In einem ersten Schritt werden die unterschiedlichen Ergebnisse in der Bewertung der Beteiligungsmöglichkeiten entlang der ersten beiden Hypothesen (1a, 1b) dargestellt. Hypothese 2 und 3 folgen im Anschluss. 


\subsection{Grundsätzliche Einstellungen zu den Beteiligungsmöglichkeiten}

Entgegen der aufgestellten Hypothese 1a und den ersten rein deskriptiven Erkenntnissen sind weibliche Ratsmitglieder gegenüber repräsentativen Beteiligungsmöglichkeiten grundsätzlich nicht signifikant skeptischer eingestellt als ihre männlichen Ratskollegen (Tab. 2). Unter dem Eindruck einer Reihe von Kontrollvariablen verschwindet der deskriptiv beobachtete Unterschied und dabei bleiben die Effekte auf der Individual- als auch auf der Kontextebene stabil. Damit kann Hypothese 1a nicht aufrechtgehalten werden. Ein Machtungleichgewicht zwischen den Geschlechtern wirkt sich also nicht grundsätzlich auf eine skeptischere Erwartungshaltung von Frauen gegenüber repräsentativen Beteiligungsmöglichkeiten aus, auch wenn ein spezifischerer Bick auf einzelnen Parteieneffekte durchaus Unterschiede aufzeigen kann (siehe Abschn. 6.2).

Robust und signifikant ist der geschlechtsspezifische Effekt hingegen bei der Bewertung partizipativer Beteiligungsmöglichkeiten: Frauen bewerten diese signifikant besser als ihre männlichen Kollegen, auch wenn auf weitere Variablen kontrolliert wird. Somit bestätigt sich zum Teil Hypothese $1 \mathrm{~b}$ und legt nahe, dass Frauen durchaus aus einem Machtungleichgewicht heraus alternative Beteiligungsmöglichkeiten gegenüber klassischen repräsentativen Verfahren präferieren. Zumindest partizipative Verfahren werden von weiblichen Ratsmitgliedern signifikant besser bewertet, wobei für direktdemokratische Beteiligungsmöglichkeiten kein signifikanter Effekt festzustellen ist. Mit Blick auf die beiden Hypothesen 1a und 1b bleiben gemischte Ergebnisse, die aber zumindest in die Richtung deuten, dass Frauen aufgrund des geschlechtsspezifischen Ungleichgewichts in ihren lokalen politischen Institutionen in alternativen Beteiligungsmöglichkeiten einen Ausweg sehen können.

Mit Blick auf die weiteren möglichen Erklärungsfaktoren fällt auf, dass vor allem Ratsmitglieder der CDU repräsentative Beteiligungsmöglichkeiten signifikant besser bewerten und direktdemokratische Beteiligungsmöglichkeiten signifikant schlechter. Direktdemokratische Beteiligungsmöglichkeiten werden darüber hinaus auch von Ratsmitgliedern der SPD schlechter bewertet. Ratsmitglieder der FDP bewerten repräsentative Beteiligungsmöglichkeiten etwas besser. Lediglich Ratsmitglieder der Linken bewerten repräsentative Beteiligungsmöglichkeiten signifikant schlechter und direktdemokratische Beteiligungsmöglichkeiten signifikant besser. Andere Parteieneffekte sind nicht festzustellen oder sind nicht robust genug, wenn Erklärungsfaktoren von der Kontextebene hinzugefügt werden. Sehr robust verhält sich dagegen der Einfluss des Effekts, den die Zugehörigkeit zur Mehrheitsfraktion für ein Ratsmitglied auf die Bewertung von repräsentativen und partizipativen Verfahren hat. Wie zu erwarten, befürworten Ratsmitglieder, die sich in der Mehrheit im Rat befinden, repräsentative Beteiligungsmöglichkeiten deutlich stärker und bewerten dafür partizipative Beteiligungsmöglichkeiten deutlich schlechter. Auch die Perzeption eines möglichen Machtverlustes hat einen Einfluss auf die Bewertung der Ratsmitglieder: Wer einen Machtverlust für den Rat befürchtet, bewertet repräsentative Beteiligungsmöglichkeiten besser und bewertet gleichzeitig partizipative und insbesondere direktdemokratische Beteiligungsmöglichkeiten deutlich schlechter. Mit dem Alter, der Amtszeit und dem Bildungsabschluss sind auch soziostrukturelle Faktoren auf der Individualebene der Befragten signifikant einflussreich. Faktoren 


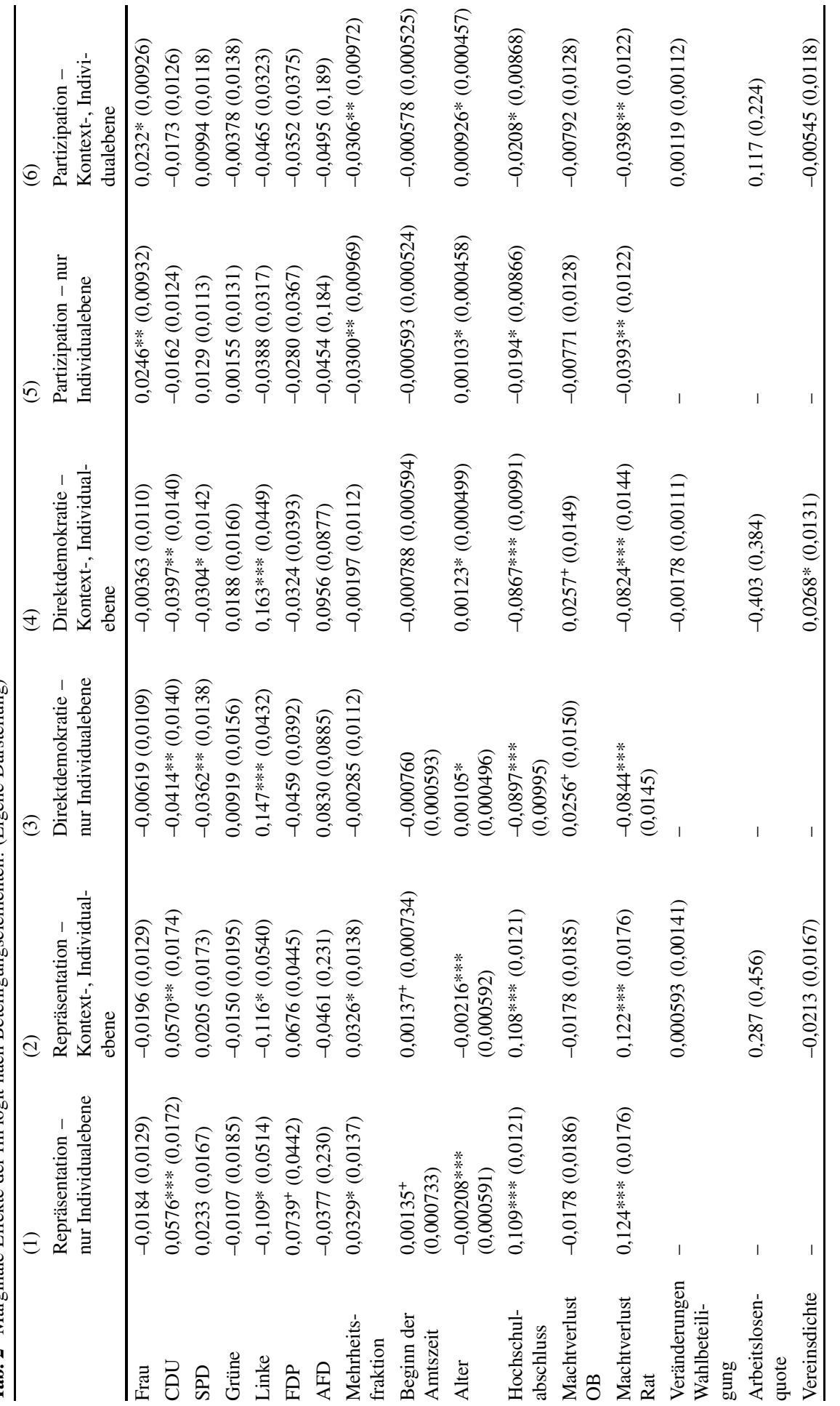




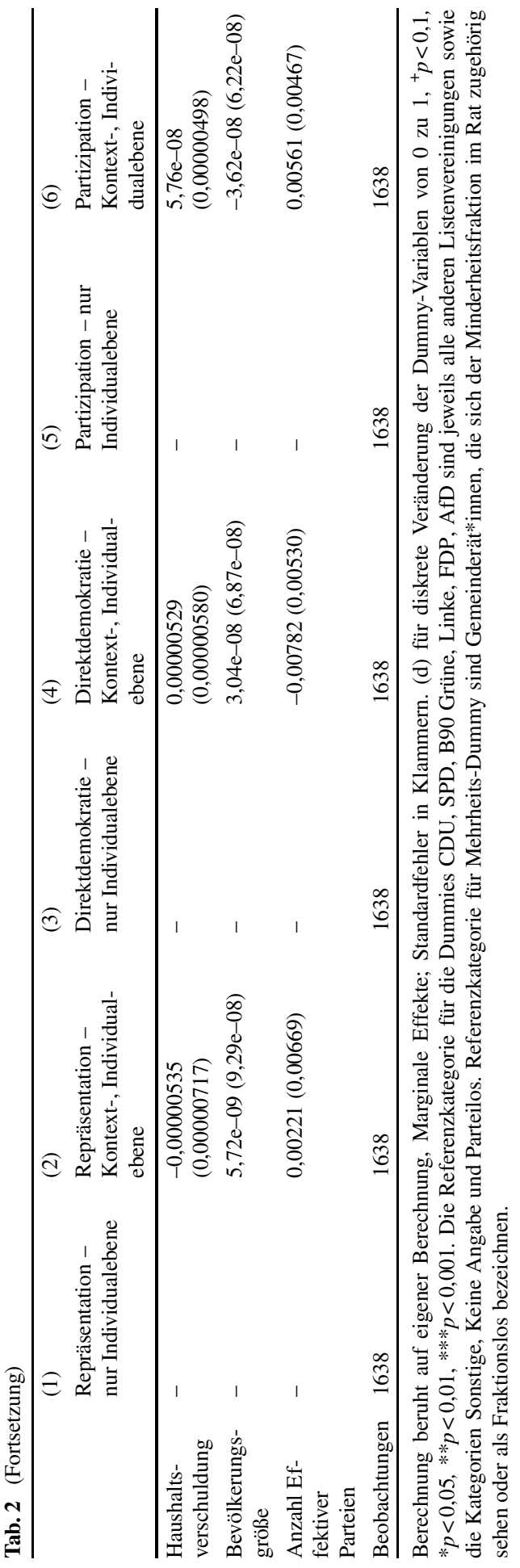


auf der Kontextualebene haben nur in einem Fall einen signifikanten Einfluss auf die Bewertung der Beteiligungsmöglichkeiten: Je höher die Vereinsdichte in einer Gemeinde, desto besser werden direktdemokratische Beteiligungsmöglichkeiten von den Befragten bewertet.

\subsection{Interaktionseffekte: Parteien und Mehrheitsposition}

Wird die Bewertung der Beteiligungsmöglichkeiten nach Geschlecht über Interaktionseffekte betrachtet, können gezielt Unterschiede zwischen weiblichen und männlichen Ratsmitgliedern bei den Erklärungsfaktoren ausgemacht werden. So werden Effekte, die über einzelne Dummyvariablen nur schwer zu identifizieren sind und feinere Zusammenhänge übersehen. Abb. 1 bis 4 bilden die Interaktionseffekte zwischen Parteien und Geschlecht über marginalen Effekte der fractional multinomial logit ab. Die marginalen Effekte als Koeffizienten geben in diesem Fall an, um wieviel Prozentpunkte sich die Bewertung einer befragten Person ändert, wenn sie beispielsweise von Nicht-CDU (0) zu CDU (1) wechselt, während gleichzeitig alle anderen Parteienvariablen konstant gehalten werden. Somit werden Vorhersagen über Interaktionen bei konstant gehaltenen Bedingungen möglich.

Schnell wird deutlich, dass bei weiblichen Ratsmitgliedern andere Erklärungsfaktoren einflussreich sind als bei ihren männlichen Ratskollegen, was für den geschlechtsspezifischen Ansatz in dieser Arbeit spricht. Gemäß dem zuvor skizzierten Machtungleichgewicht in politischen Institutionen wurde angenommen, dass Frauen andere strategische Überlegungen aufstellen als Männer das Tun und Frauen andere Entscheidungsformate suchen (müssen), um ihre Prioritäten besser durchzusetzen (Hypothese 2 und 3).

Hypothese 2 nimmt an, dass Unterschiede in der Bewertung partizipativer und direktdemokratischer Beteiligungsmöglichkeiten zwischen Männern und Frauen in rechten Parteien größer sind als in linken Parteien. H2 nimmt also eine Interaktion zwischen Geschlecht und Parteiausprägung an. Diese Hypothese lässt sich so jedoch nicht eindeutig bestätigen. Vielmehr weisen die Effekte und Unterschiede in zwei unterschiedliche Richtungen und legen somit den Schluss nahe, dass die Bewertung keinen einfachen Links-Rechts Dualismus bei den Beteiligungsmöglichkeiten folgt. So sind grundsätzlich Unterschiede zwischen Frauen und Männern bei der Partei die Linke und der CDU am größten, wobei bei der Linken die Unterschiede am stärksten ausfallen. Keine oder nur sehr geringe Unterschiede zwischen Frauen und Männern in der Bewertung lassen sich bei der SPD, bei Grünen und bei der FDP feststellen. Damit weisen vor allem die Parteien, die im baden-württembergischen Links-Rechts Vergleich den linken und rechten Rand einnehmen, Unterschiede in der Bewertung zwischen Männern und Frauen auf. Während sich die Parteien, die sich in BadenWürttemberg eher an der Mitte orientieren, in der Bewertung nicht oder wenn, nur kaum unterscheiden. ${ }^{7}$ Es kann also auch nicht grundsätzlich angenommen werden,

\footnotetext{
7 Für die AfD befinden sich im Datensatz keine weiblichen Ratsmitglieder, wenngleich die AfD im badenwürttembergischen Links-Rechts-Vergleich bundesdeutscher Parteien natürlich stark rechts einzuordnen ist.
} 

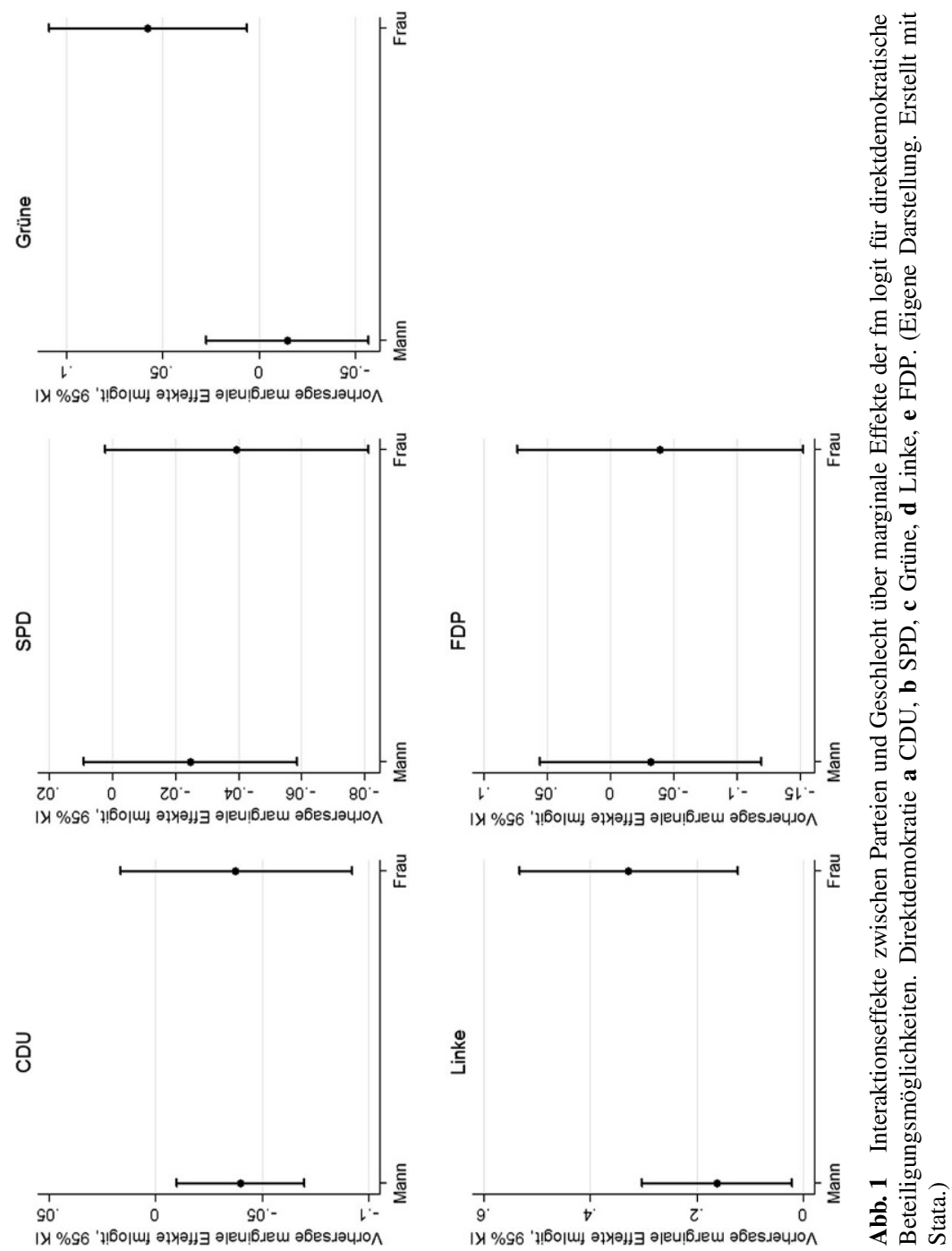

dass die Parteizugehörigkeit der Befragten in jedem Fall einen Einfluss auf deren Bewertung im Sinne des angenommenen Machtungleichgewichts hat (Abb. 1).

Abb. 4, 5 und 6 zeigen die grafische Darstellung der marginalen Effekte für die Interaktionseffekte zwischen Geschlecht und Partei auf die Bewertung der einzelnen Beteiligungsmöglichkeiten. Bei der Bewertung direktdemokratischer Beteiligungsmöglichkeiten (Abb. 4) zeigt sich, dass CDU-Männer diese signifikant schlechter bewerten, Grüne Frauen diese signifikant besser bewerten. Ratsfrauen und Ratsmänner der Linken zeigen hier aber die deutlichsten Unterschiede, während zwischen Frauen und Männern der FDP und der SPD bei direktdemokratischen Beteiligungsmöglichkeiten keine Unterschiede festzustellen sind. Damit bestätigen sich die rein deskriptiven Beobachtungen hier nur für die SPD und die Linke. Ähnliches lässt sich 


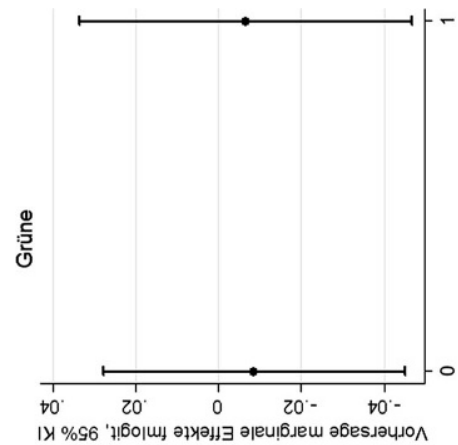

bei partizipativen Beteiligungsmöglichkeiten beobachten (Abb. 5). Wiederum sind hier zwischen SPD und FDP aber auch bei grünen Ratsmitgliedern keine signifikanten Unterschiede auszumachen. Gerade CDU-Frauen und Ratsfrauen der Linken unterscheiden sich von ihren männlichen Parteikollegen (Abb. 2).

Abb. 6 zeigt die Interaktionseffekte für repräsentative Beteiligungsmöglichkeiten und schließt damit das Beteiligungsdreieck aus repräsentativen, partizipativen und direktdemokratischen Beteiligungsmöglichkeiten, das den Befragten vorgelegt wurde. Zwar wurde für die unterschiedlichen Bewertungen von Männern und Frauen zu dieser Beteiligungsmöglichkeit keine Hypothese formuliert, sie spiegelt aber trotzdem die Ergebnisse aus den vorherigen Abb.en der marginalen Effekte unter umgekehrten Vorzeichen wieder. Wiederum sind es Frauen und Männer aus der 

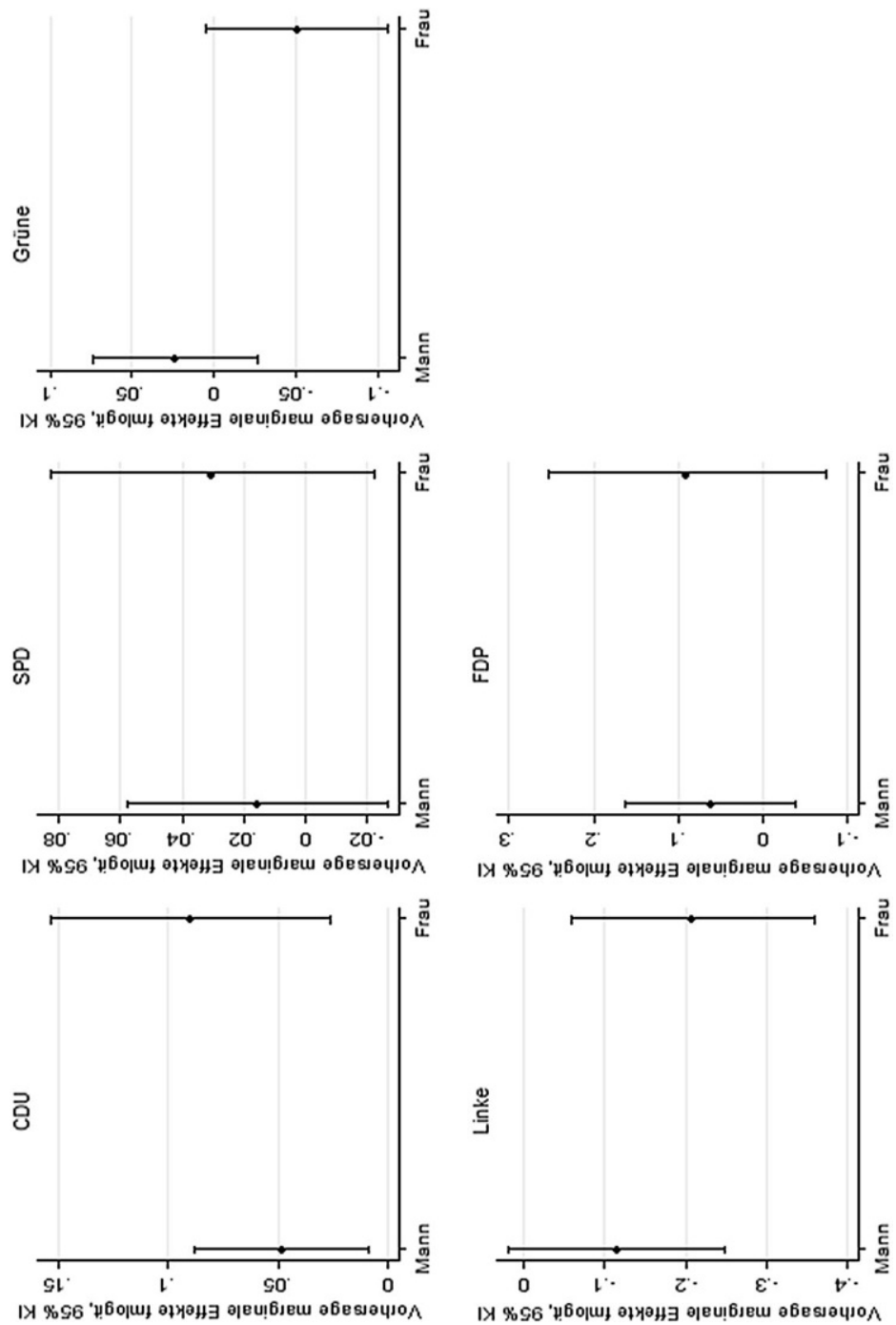

CDU und der Linken, die sich signifikant voneinander unterscheiden, während zwischen Ratsfrauen und Ratsmännern der Grünen, SPD und FDP kein signifikanter Unterschied vorliegt (Abb. 3).

Hypothese 3 nimmt an, dass die formale Zugehörigkeit von Ratsherren zur Ratsminderheit oder Ratsmehrheit im Vergleich zu ihren weiblichen Kolleginnen einen deutlich stärkeren Einfluss auf die Bewertung der unterschiedlichen Beteiligungsmöglichkeiten hat. Da Frauen sich im Rat bereits in einer strukturellen Minderheitenpositionen befinden und zusätzliche Hindernisse überwinden müssen, bevor sie ihre Präferenzen genauso wie ihre Kollegen durchsetzen können. Abb. 7 bestätigt diese Annahme für zwei der drei Beteiligungsmöglichkeiten. Männliche Ratsmitglieder, die formal der Ratsmehrheit zugehörig sind, bewerten repräsentative Betei- 



Abb. 4 Interaktionseffekte zwischen Parteien und Zugehörigkeit zur Gemeinderatsmehrheit über marginale Effekte der fm logit für repräsentative Beteiligungsmöglichkeiten. a Repräsentation, b Direktdemokratie, c Partizipation. (Eigene Darstellung. Erstellt mit Stata.)

ligungsmöglichkeiten signifikant besser und partizipative Beteiligungsmöglichkeiten signifikant schlechter. Für weibliche Ratsmitglieder ist hier kein Unterschied festzustellen. Für weibliche Ratsmitglieder macht es für ihre Bewertung also keinen Unterschied, ob sie der Minderheit oder Mehrheit im Rat angehören. Diese Ergebnisse gelten für alle Parteien und Listenvereinigungen und bei jeweils konstant gehaltenen Kontrollvariablen. Keinen signifikanten Unterschied lässt sich bei der Bewertung direktdemokratischer Beteiligungsmöglichkeiten ausmachen. Eine Erklärung dafür könnte sein, dass bei direktdemokratischen Beteiligungsmöglichkeiten die Entscheidung über eine bestimmte Sache nicht im Rat stattfindet und hiermit die Durchsetzung der jeweiligen Präferenzen unabhängig vom Geschlecht der Ratsmitglieder von diesen als sehr gering eingeschätzt werden. Die Grundsätzliche Annahme aus der Forschung zu strategischen Erwägungen von Ratsmitgliedern auf der kommunalen Ebene muss hier also angepasst werden: Auf Frauen hat ihre Zugehörigkeit zur Ratsminderheit- oder Mehrheit andere oder keinen direkten Einfluss auf die Bewertung der verschiedenen Beteiligungsmöglichkeiten (Abb. 4).

\section{Fazit}

Diese Arbeit beleuchtet eine wichtige Perspektive bei der Erforschung kommunalpolitischer Eliten, die zuvor nicht im Fokus vergleichender Studien stand und neue Erkenntnisse bringt: Den Einfluss des Geschlechts von Ratsmitgliedern auf die Bewertung lokaler politischer Beteiligung. Anhand eines umfassenden Datensatzes 
mit 1638 Befragten konnte gezeigt werden, dass die Annahmen zu institutionellen Machtungleichgewichten zwischen den Geschlechtern, klassische Erklärungsansätze aus der Einstellungsforschung zu Ratsmitgliedern ergänzen und bereichern können. Welche Erklärungsfaktoren signifikant einflussreich sind, hängt eben auch von den unterschiedlichen Erfahrungen und Perspektiven weiblicher Ratsmitglieder ab. Ratsfrauen bewerten partizipative Beteiligungsmöglichkeiten grundsätzlich besser als ihre männlichen Kollegen und auch die Kombination von Parteizugehörigkeit und Geschlecht kann einen Einfluss auf die unterschiedliche Bewertung haben. Entgegen der gängigen Forschungsliteratur leiten Frauen und Männer jedoch aus der Zugehörigkeit zur Mehrheit im Rat unterschiedliche Bewertungen für die drei Beteiligungsmöglichkeiten ab. Insgesamt sprechen die Ergebnisse dafür, dass in bestimmten Konstellationen Frauen andere strategische Entscheidungen aufgrund von Machtungleichgewichten im Rat und den Parteien treffen und sich in der Bewertung von ihren männlichen Kollegen deshalb zum Teil erheblich unterschieden. Auch wenn dies nicht immer auf alle drei getesteten Beteiligungsmöglichkeiten in gleichem Maße zutrifft. Dies spricht wiederum für den Fokus dieser Arbeit, die Einstellungen über drei unterschiedliche Beteiligungsmöglichkeiten abzubilden.

Es sind alleine die Männer im Rat, auf die ihre Zugehörigkeit zur Ratsmehrheit einen starken Einfluss auf ihre Bewertungen ausübt. Auf Frauen hat ihre Zugehörigkeit zur Gemeinderatsmehrheit im Gegensatz zu ihren männlichen Kollegen keinen Einfluss auf die Bewertung. Dabei wurde angenommen, dass Frauen sich im Rat bereits in einer Art Minderheitenposition befinden und erst zusätzliche Hindernisse überwinden müssen, bevor sie ihre Präferenzen genauso wie ihre männlichen Kollegen durchsetzen können (Shepsle 2010, S. 183-184). Die etablierte Annahme aus der Forschung zu strategischen Erwägungen von Ratsmitgliedern auf der kommunalen Ebene muss zumindest für diesen Kontext also ausdifferenziert werden (Kersting und Schneider 2016; Ruf et al. 2019; Wagschal et al. 2019): Der angenommene Effekt, Zugehörigkeit zur Mehrheit führt zu besseren Druchsetzbarkeit von Präferenzen und deshalb einer besseren Bewertung für repräsentative Beteiligungsformen und umgekehrt Zugehörigkeit zur Minderheit einer besserer Bewertung für alternative Beteiligungsformen, trifft auf weibliche Ratsmitglieder in Baden-Württemberg nicht zu. Damit verfestigt sich der Eindruck aus der Literatur von substantiellen Unterschieden zwischen Männern und Frau aufgrund von Machtungleichgewichten, wie er zum Beispiel bei Kittilson (2006) oder Erzeel und Celis (2016) beschrieben wurde.

Gemischte Ergebnisse bleiben bei der grundsätzlichen Bewertung von Frauen zu den Beteiligungsmöglichkeiten. So wirkt sich das angenommene Machtungleichgewicht im Rat zwischen den Geschlechtern nicht grundsätzlich auf eine skeptischere Erwartungshaltung von Frauen gegenüber repräsentativen Beteiligungsmöglichkeiten aus. Vor allem partizipative Beteiligungsmöglichkeiten werden von Frauen grundsätzlich besser bewertet, was damit die Annahmen von Hansen und Goenaga auf europäischer Ebene (2019, S. 6). Hansen und Geonga legen dar, dass Frauen darin einen Ausweg sehen, um ihre Präferenzen besser durchzusetzen. Dies bleibt für den baden-württembergischen Fall allerdings nur eine naheliegende Vermutung und über das genaue Motiv könnten qualitative Interviews ergänzende Erkenntnisse liefern. Unterschiede werden auch bei einem näheren Blick auf Interaktionen zwischen 
dem Geschlecht der Befragten und ihrer Parteizugehörigkeit sichtbar. Hier wurde der Parteizugehörigkeit ein moderierender Effekt auf die Bewertung von Frauen zugeschrieben, der in rechten Parteien stärkere Unterschiede zwischen Männern und Frauen hervorrufen sollte. So konnte dieser Effekt zwar bei der CDU festgestellt werden, gilt aber auch für die Partei Die Linke. Somit sind es die Parteien am rechten und am linken Rand, bei denen die Unterschiede in der Bewertung zwischen Männern und Frauen am größten sind, während sich Männer und Frauen in Parteien, die sich in Baden-Württemberg eher an der Mitte orientieren (Grüne, SPD, FDP), sich in der Bewertung deutlich weniger oder nicht unterscheiden. Ergebnisse aus anderen Länder- und Parteiensystemkontexten wie von Campbell und Childs (2015) und Barnes und Cassese (2017) können so also nicht eins zu eins übertragen werden. Allerdings ist der moderierende Effekt nicht nur zwischen den verschiedenen Parteien, sondern auch innerhalb zwei bestimmter Parteien durchaus auch in BadenWürttemberg zu erkennen, eben nur ganz links und ganz rechts. Eine Erklärung kann hier in dieser Arbeit dafür nicht geliefert werden, sollte aber weiter untersucht werden. Natürlich haben neben der Parteizugehörigkeit auch andere Effekte einen (zum Teil sehr starken) Einfluss auf die Bewertung der Ratsfrauen und Ratsmännern, wie ihr Alter, ein Hochschulabschluss, die individuelle Amtszeit, aber auch die Perzeption eines möglichen Machtverlusts im Rat und zum Teil auch auf der Kontextebene die Vereinsdichte, wie diese auch verschiedene Autor*innen zuvor feststellen konnten (Heinelt 2013c; Kersting und Schneider 2016; Ruf et al. 2019; Wagschal et al. 2019).

Mit Hilfe des Datensatzes und des speziellen methodischen Vorgehens können auch wichtige Erkenntnisse für die methodische Herangehensweise gezogen werden. So ist durchaus nicht irrelevant, die drei unterschiedlichen Beteiligungsformen über drei abhängigen Variablen zu operationalisieren und diese dann auch ins Verhältnis zu setzen, da sonst vor allem Unterschiede zwischen partizipativen und direktdemokratischen Beteiligungsformen übersehen werden. Darüber hinaus ist deutlich geworden, wie wichtig das Geschlecht als Analysekategorie in der Erforschung der Einstellung kommunalpolitischer Eliten sein kann. Gerade mit Blick auf die wachsende Zahl von Frauen in politischen Ämtern und ihrer zunehmenden Bedeutung als Gatekeeper bei politischen Entscheidungen, sind ihre Einstellungen, ihre strategischen Überlegungen hochrelevant. Unterschiedliche Repräsentationsniveaus von Frauen in den kommunalpolitischen Institutionen, Parteien und zwischen den Bundesländern versprechen darüber hinaus weitere interessante Schlussfolgerungen dazu, welche Effekte Parteien, die Institution des Rates oder auch ganze kommunalpolitische Systeme auf die strategischen Überlegungen von weiblichen politischen Eliten haben (können). Die Analyse zeigt aber auch, dass hier noch viel Unschärfe existiert: Warum weibliche Ratsmitglieder genau in partizipativen Beteiligungsformen einen Ausweg sehen um ihre Präferenzen durchzusetzen, nicht aber in direktdemokratischen Beteiligungsformen, sollte weiter untersucht werden. In einem nächsten Schritt könnten dafür auch mit qualitativen Methoden die Motive stärker herausgearbeitet werden. Ein erster Ansatzpunkt könnte der Grad an Einfluss sein, den Ratsmitglieder auf die drei unterschiedlichen Beteiligungsformen ausüben können. Eventuell nehmen sie an, dass sie bei partizipativen Beteiligungsformen viel besser ihre Interessen durchsetzen können als bei direktdemokratischen 
Beteiligungsformen. Das ist aber nur eine der möglichen Spekulationen und welche weiteren strategischen Überlegungen vielleicht hinter diesen Einstellungen liegen, konnte auch diese Arbeit nur vereinzelt ausleuchten. Sicher ist, strategische Perspektiven auf die Einstellung von Ratsmitgliedern und ihre geschlechtsspezifischen Perspektiven bleiben ein lohnenswertes Feld.

Funding Open Access funding enabled and organized by Projekt DEAL.

Open Access Dieser Artikel wird unter der Creative Commons Namensnennung 4.0 International Lizenz veröffentlicht, welche die Nutzung, Vervielfältigung, Bearbeitung, Verbreitung und Wiedergabe in jeglichem Medium und Format erlaubt, sofern Sie den/die ursprünglichen Autor(en) und die Quelle ordnungsgemäß nennen, einen Link zur Creative Commons Lizenz beifügen und angeben, ob Änderungen vorgenommen wurden.

Die in diesem Artikel enthaltenen Bilder und sonstiges Drittmaterial unterliegen ebenfalls der genannten Creative Commons Lizenz, sofern sich aus der Abbildungslegende nichts anderes ergibt. Sofern das betreffende Material nicht unter der genannten Creative Commons Lizenz steht und die betreffende Handlung nicht nach gesetzlichen Vorschriften erlaubt ist, ist für die oben aufgeführten Weiterverwendungen des Materials die Einwilligung des jeweiligen Rechteinhabers einzuholen.

Weitere Details zur Lizenz entnehmen Sie bitte der Lizenzinformation auf http://creativecommons.org/ licenses/by/4.0/deed.de.

Interessenkonflikt F. Ruf gibt an, dass kein Interessenkonflikt besteht.

\section{Literatur}

Aitchison, J. 2003. The statistical analysis of compositional data. Caldwell: Blackburn Press.

Bäck, Hanna, Marc Debus, und Jochen Müller. 2014. Who takes the parliamentary floor? The role of gender in speech-making in the Swedish Riksdag. Political Research Quarterly 67(3):504-518. https://doi. org/10.1177/1065912914525861.

Baekgaard, Martin, und Ulrik Kjaer. 2012. The gendered division of labor in assignments to political committees: discrimination or self-selection in Danish local politics? Politics \& Gender 8(04):465-482. https://doi.org/10.1017/S1743923X12000499.

Barnes, Tiffany D., und Erin C. Cassese. 2017. American party women: a look at the gender gap within parties. Political Research Quarterly 70(1):127-141. https://doi.org/10.1177/1065912916675738.

Bengtsson, Åsa, und Mikko Mattila. 2009. Direct democracy and its critics: support for direct democracy and 'stealth' democracy in Finland. West European Politics 32(5):1031-1048. https://doi.org/10. 1080/01402380903065256.

Blome, Agnes, und Gesine Fuchs. 2017. Macht und substantielle Repräsentation von Frauen. FEMINA POLITICA - Zeitschrift für feministische Politikwissenschaft 26(1):55-69. https://doi.org/10.3224/ feminapolitica.v26i1.04.

Bräuninger, Thomas, und Marc Debus. 2012. Parteienwettbewerb in den deutschen Bundesländern Unter Mitarbeit von Jochen Müller. Wiesbaden: VS. https://doi.org/10.1007/978-3-531-93226-2.

Brunsbach, Sandra. 2011. Machen Frauen den Unterschied? Parlamentarierinnen als Repräsentantinnen frauenspezifischer Interessen im Deutschen Bundestag. Zeitschrift für Parlamentsfragen 42(1):3-24. https://doi.org/10.5771/0340-1758-2011-1-3.

Buis, Maarten L. 2008. FMLOGIT: Stata module fitting a fractional multinomial logit model by quasi maximum likelihood. Stata. Boston College Department of Economics.

Butler, Daniel M., und Jessica Robinson Preece. 2016. Recruitment and perceptions of gender bias in party leader support. Political Research Quarterly 69(4):842-851. https://doi.org/10.1177/ 1065912916668412.

Campbell, Rosie, und Sarah Childs. 2015. 'To the left, to the right': representing conservative women's interests. Party Politics 21(4):626-637. https://doi.org/10.1177/1354068813491536. 
Celis, Karen, und Sarah Childs. 2012. The substantive representation of women: what to do with conservative claims? Political Studies 60(1):213-225. https://doi.org/10.1111/j.1467-9248.2011.00904. $\mathrm{X}$.

Celis, Karen, Sarah Childs, und Johanna Kantola. 2016. Regendering party politics: an introduction. Party Politics 22(5):571-575. https://doi.org/10.1177/1354068816655567.

Chaney, Paul. 2014. The substantive representation of women-Does issue-salience matter? Party politicization and UK Westminster elections 1945-2010. The British Journal of Politics and International Relations 16(1):96-116. https://doi.org/10.1111/1467-856X.12007.

Childs, Sarah. 2010. Women and British party politics: descriptive, substantive and symbolic representation. London: Routledge.

Childs, Sarah, und Mona Lena Krook. 2009. Analysing women's substantive representation: from critical mass to critical actors. Government and Opposition 44(02):125-145. https://doi.org/10.1111/j.14777053.2009.01279.x.

Childs, S.L., und M.C. Kittilson. 2016. Feminizing political parties: womens party member organizations within European parliamentary parties. Party Politics 22(5):598-608. https://doi.org/10.1177/ 1354068816654320.

Childs, Sarah, und Joni Lovenduski. 2013. Political Representation. Oxford University Press. http:// oxfordhandbooks.com/view/10.1093/oxfordhb/9780199751457.001.0001/oxfordhb-9780199751457e-19. Zugegriffen: 1. Febr. 2020.

Copus, Colin. 2010. The councillor: governor, governing, governance and the complexity of citizen engagement. The British Journal of Politics and International Relations 12(4):569-589. https://doi.org/ 10.1111/j.1467-856X.2010.00423.x.

Debus, Marc, und Martin Gross. 2016. Coalition formation at the local level: institutional constraints, party policy conflict, and office-seeking political parties. Party Politics 22(6):835-846. https://doi.org/10. 1177/1354068815576292.

Debus, Marc, Christoph Knill, und Jale Tosun. 2012. Drum zahle, wer sich ewig bindet: Eine Analyse der Gebührenhöhe für eingetragene Lebenspartnerschaften in Baden-Württemberg. Politische Vierteljahresschrift 53(1):1-28. https://doi.org/10.5771/0032-3470-2012-1-1.

Denters, Bas, und Pieter-Jan Klok. 2013. Citizen democracy and the responsiveness of councillors: the effects of democratic institutionalisation on the role orientations and role behaviour of councillors. Local Government Studies 39(5):661-680. https://doi.org/10.1080/03003930.2012.670747.

Egner, Björn. 2013a. Das Ratsmitglied, das unbekannte Wesen. In Das deutsche Gemeinderatsmitglied: Problemsichten - Einstellungen - Rollenverständnis, Hrsg. Björn Egner, Max-Christopher Krapp, und Hubert Heinelt, 57-67. Wiesbaden: Springer. https://doi.org/10.1007/978-3-531-94361-9_3.

Egner, Björn (Hrsg.). 2013b. Local councillors in Europe. Wiesbaden: Springer VS.

Egner, Björn, Max-Christopher Krapp, und Hubert Heinelt. 2013a. Das deutsche Gemeinderatsmitglied. Wiesbaden: Springer VS. abrufbar unter: http://site.ebrary.com/lib/alltitles/docDetail.action? docID $=10624955$.

Egner, Björn, Max-Christopher Krapp, und Hubert Heinelt. 2013b. Das deutsche Gemeinderatsmitglied Problemsichten - Einstellungen - Rollenverständnis. Wiesbaden: Springer. https://doi.org/10.1007/ 978-3-531-94361-9.

Erzeel, S., und K. Celis. 2016. Political parties, ideology and the substantive representation of women. Party Politics 22(5):576-586. https://doi.org/10.1177/1354068816655561.

Font, Joan, und Carolina Galais. 2011. The qualities of local participation: the explanatory role of ideology, external support and civil society as organizer: local participation in Catalonia, Spain. International Journal of Urban and Regional Research https://doi.org/10.1111/j.1468-2427.2011.01018.x.

Funk, P., und C. Gathmann. 2015. Gender gaps in policy making: evidence from direct democracy in Switzerland. Economic Policy 30(81):141-181. https://doi.org/10.1093/epolic/eiu003.

Gabriel, Oscar W., und Norbert Kersting. 2014. Politische Beteiligung und lokale Demokratie. In Partizipation im Wandel. Unsere Demokratie zwischen Wählen, Mitmachen und Entscheiden, Hrsg. Bertelsmann-Stiftung, Baden-Württemberg Stiftung, 1-140. Gütersloh: Bertelsmann Stiftung.

Gross, Martin. 2017. Machtstrukturen in der lokalen Politik. In Politische Einstellungen von Kommunalpolitikern im Vergleich, Hrsg. Markus Tausendpfund, Angelika Vetter, 111-149. Wiesbaden: Springer. https://doi.org/10.1007/978-3-658-16398-3_4.

Gross, Martin, und Michael Jankowski. 2018. Party competition in multilevel democracies. Evidence from the local manifesto project. Working paper.

Hansen, Michael A., und Agustín Goenaga. 2019. Gender and democratic attitudes: do women and men prioritize different democratic institutions? Politics \& Gender https://doi.org/10.1017/ S1743923X19000473. 
Hazan, Reuven Y., und Gid Rahat. 2010. Democracy within parties: candidate selection methods and their political consequences. Oxford, New York: Oxford University Press.

Heinelt, Hubert. 2013a. Councillors and democracy: what do they think, and how can differences in their views be explained? In Local councillors in Europe, Hrsg. Björn Egner, David Sweeting, und PieterJan Klok, 85-96. Wiesbaden: Springer. https://doi.org/10.1007/978-3-658-01857-3_5.

Heinelt, Hubert. 2013b. Councillors' notions of democracy, and their role perception and behaviour in the changing context of local democracy. Local Government Studies 39(5):640-660. https://doi.org/10. 1080/03003930.2012.670746.

Heinelt, Hubert. 2013c. Welches Demokratieverständnis haben deutsche Ratsmitglieder und wie schlägt es sich in ihren Handlungsorientierungen nieder? In Das deutsche Gemeinderatsmitglied. problemsichten. Einstellungen. Rollenverständnis, Hrsg. Björn Egner, Max-Christopher Krapp, und Hubert Heinelt, 105-126. Wiesbaden: Springer VS.

Hoecker, Beate. 1995. Politische Partizipation von Frauen: Kontinuität und Wandel des Geschlechterverhältnisses in der Politik; ein einführendes Studienbuch. Opladen: Leske + Budrich.

Holtkamp, Lars. 2006. Parteien und Bürgermeister in der Repräsentativen Demokratie. Kommunale Konkordanz- und Konkurrenzdemokratie im Vergleich. Politische Vierteljahresschrift 47(4):641-661. https://doi.org/10.1007/s11615-006-0345-y.

Holtkamp, Lars, Thomas Bathge, und Caroline Friedhoff. 2015. Kommunale Parteien und Wählergemeinschaften in Ost- und Westdeutschland. Zeitschrift für Vergleichende Politikwissenschaft 9(1-2):1-18. https://doi.org/10.1007/s12286-015-0243-z.

Holtkamp, Lars, Sonja Schnittke, und Elke Wiechmann. 2011. Die Stagnation der parlamentarischen Frauenrepräsentanz - Erklärungsansätze am Beispiel deutscher Großstädte. Zeitschrift für Parlamentsfragen 42(1):35-49. https://doi.org/10.5771/0340-1758-2011-1-35.

Hornig, Eike-Christian. 2011. Die Parteiendominanz direkter Demokratie in Westeuropa. Nomos. http:// www.nomos-elibrary.de/index.php?doi=10.5771/9783845228808. Zugegriffen: 8. Nov. 2020.

Karlsson, Martin. 2012. Participatory initiatives and political representation: the case of local councillors in Sweden. Local Government Studies 38(6):795-815. https://doi.org/10.1080/03003930.2012.688036.

Kenny, Meryl. 2007. Gender, institutions and power: a critical review. Politics 27(2):91-100. https://doi. org/10.1111/j.1467-9256.2007.00284.x.

Kenny, Meryl, und Tània Verge. 2016. Opening up the black box: gender and candidate selection in a new era. Government and Opposition 51(03):351-369. https://doi.org/10.1017/gov.2016.5.

Kersting, Norbert, und Sebastian H. Schneider. 2016. Neue Machtansprüche in der Kommunalpolitik. Zeitschrift für Vergleichende Politikwissenschaft 10(3-4):311-339. https://doi.org/10.1007/s12286-0160308-7.

Kim, Jeong Hyun. 2019. Direct democracy and women's political engagement. American Journal of Political Science 63(3):594-610. https://doi.org/10.1111/ajps.12420.

King, Gary, Robert O. Keohane, und Sidney Verba. 1994. Designing social inquiry: scientific inference in qualitative research. Princeton: Princeton University Press.

Kittilson, Miki Caul. 2006. Challenging parties, changing parliaments: women and elected office in contemporary Western Europe. Columbus: Ohio State University Press.

Kost, Andreas, und Hans-Georg Wehling. 2010. Kommunalpolitik in den deutschen Ländern Eine Einführung. Wiesbaden: VS. https://doi.org/10.1007/978-3-531-92034-4.

Krook, Mona Lena, und Pippa Norri. 2014. Beyond quotas: strategies to promote gender equality in elected office. Political Studies 62(1):2-20. https://doi.org/10.1111/1467-9248.12116.

Krummenauer, Eva, Florian Ruf, und Uwe Wagschal. 2019. Stimmen Sie mit Ja!: Eine qualitative Inhaltsanalyse der öffentlichen Kommunikation zu sechs Bürgerentscheiden. In Demokratie-Monitoring Baden-Württemberg 2016/2017, Hrsg. Baden-Württemberg Stiftung, 293-321. Wiesbaden: Springer. https://doi.org/10.1007/978-3-658-23331-0_12.

Lawless, Jennifer L., und Richard Logan Fox. 2005. It takes a candidate: why women don't run for office. Cambridge, New York: Cambridge University Press.

Leininger, Arndt. 2015. Popular support for DirectDemocracy in Europe. In ECPR joint sessions, Warschau. https://ecpr.eu/Filestore/PaperProposal/81025c54-6d96-42eb-99bd-d1c6769be3ac.pdf. Zugegriffen: 10. Nov. 2021.

Lovenduski, Joni. 2005. Feminizing politics. Cambridge, Malden: Polity Press.

Lowndes, Vivien, Lawrence Pratchett, und Gerry Stoker. 2006. LOCAL POLITICAL PARTICIPATION: THE IMPACT OF RULES-IN-USE. Public Administration 84(3):539-561. https://doi.org/10.1111/ j.1467-9299.2006.00601.x.

Magin, Raphael. 2011. Die geringere Hälfte: Erscheinungsformen, Entwicklungen und Ursachen der Unterrepräsentation von Frauen in deutschen Parlamenten. Berlin: Lit. 
Mckenna, Dave. 2012. Local politicians' attitudes towards participatory initiatives: a Bulpittian perspective. Public Money \& Management 32(2):103-110. https://doi.org/10.1080/09540962.2012.656012.

Mielke, Gerd, und Ulrich Eith. 1994. Honoratioren oder Parteisoldaten?: eine Untersuchung der Gemeinderatskandidaten bei der Kommunalwahl 1989 in Freiburg, 1. Aufl., Bochum: Universitätsverlag N. Brockmeyer.

Mullahy, John. 2010. Multivariate fractional regression estimation of econometric share models. Cambridge: National Bureau of Economic Research. http://www.nber.org/papers/w16354.pdf.

Norris, Pippa. 2004. Electoral engineering: voting rules and political behavior. Cambridge, New York: Cambridge University Press.

Norris, Pippa, Elizabeth Vallance, und Joni Lovenduski. 1992. Do candidates make a difference? Gender, race, ideology and incumbency. Parliamentary Affairs 45(4):496-517. https://doi.org/10.1093/ oxfordjournals.pa.a052379.

Nyhuis, Dominic. 2016. Partei oder Person? Parteispezifische Wahlmotive bei baden-württembergischen Kommunalwahlen. Zeitschrift für Parlamentsfragen 47(3):657-669. https://doi.org/10.5771/03401758-2016-3-657.

Nyhuis, Dominic. 2017. Estimating policy positions of local parties in elections with multi-vote ballots. Local Government Studies 43(3):475-498. https://doi.org/10.1080/03003930.2017.1300146.

Papke, Leslie E., und Jeffrey M. Wooldridge. 1996. Econometric methods for fractional response variables with an application to $401(\mathrm{~K})$ plan participation rates. Journal of Applied Econometrics 11(6):619-632.

Phillips, Anne. 1994. Dealing with difference: a politics of ideas or a politics of presence? Constellations 1(1):88-91. https://doi.org/10.1111/j.1467-8675.1994.tb00005.x.

Phillips, Anne. 1995. The politics of presence. Oxford,: New York: Clarendon Press, Oxford University Press.

Pitkin, Hanna Fenichel. 1967. The concept of representation, 1. Aufl., Berkeley: Univ. of California Press.

Pruysers, Scott, und Julie Blais. 2017. Why won't Lola run? An experiment examining stereotype threat and political ambition. Politics \& Gender 13(02):232-252. https://doi.org/10.1017/ S1743923X16000544.

Ruf, Florian. 2019. Does non-quota strategy matter? A comparative study on candidate selection and women's representation at the local level in Germany. Politics \& Gender https://doi.org/10.1017/ S1743923X18001083.

Ruf, Florian, Eva Krummenauer, und Uwe Wagschal. 2019. Machtlos durch Bürgerbeteiligung? In Demokratie-Monitoring Baden-Württemberg 2016/2017: Studien zu Demokratie und Partizipation, 273-292. Wiesbaden: Springer. https://doi.org/10.1007/978-3-658-23331-0_11.

Schwarzwälder Bote. 2020. Rathauschefinnen vernetzen sich. Schwarzwälder Bote. https://www. schwarzwaelder-bote.de/inhalt.zimmern-rathauschefinnen-vernetzen-sich.333a83fe-d735-45f5-beb40d4f62f1b526.html. Zugegriffen: 10. Nov. 2021.

Schwindt-Bayer, Leslie A. 2006. Still supermadres? Gender and the policy priorities of Latin American legislators. American Journal of Political Science 50(3):570-585. https://doi.org/10.1111/j.1540-5907. 2006.00202.x.

Shepsle, Kenneth A. 2010. Analyzing politics: rationality, behavior, and institutions, 2. Aufl., New York: W.W. Norton.

Smith, Adrienne R., Beth Reingold, und Michael Leo Owens. 2012. The political determinants of women's descriptive representation in cities. Political Research Quarterly 65(2):315-329. https://doi.org/10. $1177 / 1065912910395327$.

Snyder, Richard. 2001. Scaling down: the subnational comparative method. Studies in Comparative International Development 36(1):93-110. https://doi.org/10.1007/BF02687586.

Stecker, Christian. 2017. Parteien und Fraktionsgeschlossenheit auf der kommunalen Ebene. In Politische Einstellungen von Kommunalpolitikern im Vergleich, Hrsg. Markus Tausendpfund, Angelika Vetter, 323-348. Wiesbaden: Springer. https://doi.org/10.1007/978-3-658-16398-3_10.

Sundström, Aksel, und Daniel Stockemer. 2015. What determines women's political representation at the local level? A fine-grained analysis of the European regions. International Journal of Comparative Sociology 56(3):254-274. https://doi.org/10.1177/0020715215595691.

Tausendpfund, Markus, und Angelika Vetter. 2017. Politische Einstellungen von Kommunalpolitikern im Vergleich. http://search.ebscohost.com/login.aspx direct $=$ true $\&$ scope $=$ site $\& d b=n l e b k \& d b=n l a b k \&$ AN=1510950. Zugegriffen: 23. Mai 2017.

Verge, Tánia, und Sylvia Claveria. 2016. Gendered political resources: the case of party office. Party Politics https://doi.org/10.1177/1354068816663040. 
Vetter, Angelika (Hrsg.). 2008. Erfolgsbedingungen lokaler Bürgerbeteiligung, 1. Aufl., Wiesbaden: VS. abrufbar unter: http://gso.gbv.de/DB=2.1/PPNSET?PPN=571013007.

Vetter, Angelika. 2017. Beteiligungskulturen und Bürgerbeteiligung. In Politische Einstellungen von Kommunalpolitikern im Vergleich, Hrsg. Markus Tausendpfund, Angelika Vetter, 415-456. Wiesbaden: Springer VS.

Wagschal, Uwe, Florian Ruf, und Eva Krummenauer. 2019. Repräsentative, direktdemokratische und partizipative Beteiligung: Die Einstellung von Bürgermeistern zur lokalen politischen Beteiligung in Baden-Württemberg. Zeitschrift für Vergleichende Politikwissenschaft 13(1):33-59. https://doi.org/ 10.1007/s12286-018-0401-1.

Wängnerud, Lena. 2009. Women in parliaments: descriptive and substantive representation. Annual Review of Political Science 12(1):51-69. https://doi.org/10.1146/annurev.polisci.11.053106.123839. 